\title{
SOME SPECTRAL SEQUENCES ASSOCIATED WITH FIBRATIONS
}

\author{
BY \\ ELDON DYER AND DANIEL S. KAHN
}

The object of this paper is to establish certain spectral sequences, analogous to some discovered by Eilenberg and Moore, for the class of Dold fibrations and for a wide class of homology theories.

In writing this paper we have adopted the language and techniques of spectra. This is perhaps an interim measure as recent work of Boardman, Heller, Puppe, and others indicates the correct setting for these considerations may be some stable category. However, that is work in progress and whatever its resolution, it appears likely the arguments employed here will hold without substantial modification in that category.

A description is given (details to appear in [7]) of a process by which each Dold fibration can be shown to be fiber homotopy equivalent to an associated bundle to a principal bundle. This is done with sufficient flexibility to incorporate the classical principal bundle theory and results obtained apply thereto.

The homology theories included are those arising from multiplicative spectra for which it is possible to obtain resolutions by spectra yielding projective resolutions of $h_{*}(F)$ over $h_{*}(G)$ where $F$ is a right $G$-space, $G$ being a topological monoid. In this paper these resolutions are obtained geometrically either through the use of strictly associative ring spectra or by a device discovered by Atiyah [1]. Thus, the spectral sequences obtained are valid for homology theories including ordinary homology with coefficients in a ring, stable homotopy, various bordism theories, and $K$-theory.

The first mentioned device for obtaining these resolutions relies heavily on the notion of strict associativity (see Definition 2.7). This is required in our constructions by the fact that while one has arbitrary pushouts in the topological category, in the associated homotopy category one has only weak pushouts. These do not suffice for the constructions used here.

The paper is organized into five sections:

$\S$ I. Point set topological background.

§II. Spectra.

§III. Resolutions of spectra.

$\S I V$. The spectral sequence.

$\S \mathrm{V}$. Application to fibrations.

Received by the editors August 20, 1968. 
Within each section, all theorems, definitions, etc. are numbered sequentially. Thus Definition (2.33) is the thirty-third numbered item of §II.

I. Point set topological background. For ease of exposition we have elected to work in the category of quasi-topological spaces and mappings. For basic definitions and properties of this category the reader is referred to Spanier [11]. For a more complete development and certain results used specifically herein the reader is referred to the forthcoming book by Dyer and Eilenberg [7].

From the topological viewpoint there is no loss in working in this category as there is a functor from the category of topological spaces to it under which the notion of continuous map with domain a compact Hausdorff space is unchanged. Thus homotopy groups and the other singular algebraic functors are unchanged. Also, this functor imbeds the category of Hausdorff $k$-spaces [13] as a full subcategory of the quasi-topological category. Thus if one checks that decomposition spaces obtained by constructions of this paper, when obtained from Hausdorff $k$-spaces, yield again such spaces, the theorems and proofs herein restrict, unaltered, to that smaller category.

In this section we give a brief resumé of definitions and results in the category $Q$ of quasi-topological spaces and mappings used later. Few proofs are included as they can be found elsewhere [7]. We drop the prefix quasi with the understanding that we consider only the category $Q$ and categories derived from it.

Definition (1.1). A topological monoid is a space $H$ together with a continuous product

$$
\mu: H \times H \rightarrow H,
$$

hereafter written $\mu(g, h)=g h$, and a unit $e \in H$, such that

$$
(g h) i=g(h i), \quad \text { for }(g, h, i) \in H \times H \times H,
$$

and

$$
e g=g=g e, \text { for } g \in H .
$$

For a topological monoid $H$, a left $H$-space is a pair $(X, a)$ consisting of a space $X$ and a mapping $a: H \times X \rightarrow X$, hereafter written $a(g, x)=g x$, such that

$$
(g h) x=g(h x), \text { for }(g, h) \in H \times H \text { and } x \in X,
$$

and

$$
e x=x, \text { for } x \in X .
$$

For left $H$-spaces $(X, a)$ and $\left(X^{\prime}, a^{\prime}\right)$, the mapping $f: X \rightarrow X^{\prime}$ is a map of left $H$-spaces provided

$$
f g x=g f x, \text { for } g \in H \text { and } x \in X .
$$

The category $H Q$ has left $H$-spaces as objects and maps of left $H$-spaces as morphisms. The category $Q H$ is similarly defined for right actions by $H$. 
The category $Q$ has the property that for $X$ an object of $Q, B$ a set and $p: X \rightarrow B$ an onto function, there exists a unique quasi-topology on $B$ such that $p$ is a projection in the category $Q$; i.e., for $Y$ an object of $Q$ and $g: B \rightarrow Y$ a function, $g$ is a morphism of $Q$ if and only if $g p$ is a morphism of $Q$. In this case we say $B$ has the quotient space topology (of the equivalence relation defined by $p$ ).

Definition (1.2). For $X$ and $Y$ objects of $Q H$ and $H Q$, respectively, let $\sim$ be the equivalence relation generated by the relation $(x h, y) \sim(x, h y)$, and let $X \times_{H} Y$ denote the space of equivalence classes so defined with the quotient space topology of $\sim: X \times Y \rightarrow X \times{ }_{H} Y$.

Then $\times_{H}$ defines a covariant functor

$$
\times_{H}: Q H \times H Q \rightarrow Q .
$$

The functions $l_{X}: X \rightarrow X \times H$ and $\tilde{r}_{Y}: Y \rightarrow H \times Y$, defined by $l_{X}(x)=(x, e)$ and $\tilde{r}_{Y}(y)=(e, y)$, determine homeomorphisms

$$
l_{X}: X \rightarrow X \times_{H} H \text { and } r_{Y}: Y \rightarrow H \times_{H} Y .
$$

If $X$ and $X^{\prime}$ are objects of $Q H$, then $X \times X^{\prime}$ is an object of $Q H$ with action $\left(x, x^{\prime}\right) h=\left(x h, x^{\prime} h\right)$. Trivial action imbeds $Q$ as a full subcategory of $Q H$. For $F$, $X$ and $Y$ objects of $Q, Q H$ and $H Q$, respectively,

$$
(F \times X) \times_{H} Y \cong F \times\left(X \times_{H} Y\right),
$$

where $\cong$ denotes "is homeomorphic to."

For $A$ and $X$ objects of $Q$ and $Q H$, respectively, the space maps of $A$ to $X$, written $(A, X)$, is given the structure of a right $H$-space by defining an action

$$
a:(A, X) \times H \rightarrow(A, X)
$$

by $a(f, h) c=(f c) h$, for $f \in(A, X), h \in H$ and $c \in A$. Thus,

$$
\text { ( , ): } Q^{o p} \times Q H \rightarrow Q H
$$

is a covariant functor. For $c \in A, \eta_{c}:(A, X) \rightarrow X$ is the evaluation map $\eta_{c} f=f c$. If $X$ is an object of $Q H$, then $\eta_{c}$ is a $Q H$-morphism. For $c \in A, \eta_{c}$ is a natural transformation of $(A$,$) into the identity functor of Q H$.

For a space $X$, the path space $P X$ of $X$ is the subspace of $\left(R^{+}, X\right) \times R^{+}$of all pairs $(f, l)$ such that $f t=f l$ for $l \leqq t$, where $R^{+}$denotes the nonnegative real numbers. Maps $\eta_{0}: P X \rightarrow X$ and $\eta_{\tau}: P X \rightarrow X$ are defined by

$$
\eta_{0}(f, l)=f o \text { and } \eta_{\tau}(f, l)=f l \text {. }
$$

For a map $p: X \rightarrow B$ let $W^{p}$ be the subspace of $X \times P B$ of all pairs $(x,(f, l))$ with $p x=\eta_{0} f$. The map $p$ is a (strong) fibration if there are maps called path lifting function and delay function

$$
\Lambda^{p}: W^{p} \rightarrow P X \quad \text { and } \quad \sigma^{p}: W^{p} \rightarrow R^{+}
$$

with

$$
\eta_{0} \Lambda^{p}(x,(f, l))=x \text { and } p \Lambda^{p}(x,(f, l))(t+s)=f t,
$$


for $s=\sigma^{p}(x,(f, l))$ and $t \in R^{+}$(and $\left.\sigma^{p}\left(W^{p}\right)=0\right)$. \{This notion of fibration is due to Dold and that of strong fibration is due to Hurewicz.\}

If $X$ is an object of $Q H$ and $p x=p x h$ for all $(x, h) \in X \times H$, then $X \times P B, P X$ and $W^{p}$ are objects of $Q H$. If, with the trivial action of $H$ on $R^{+}$, both $\Lambda^{p}$ and $\sigma^{p}$ are $Q H$-morphisms, then $p$ is said to be a (strong) right $H$-fibration. It is clear that a (strong) right $H$-fibration is a (strong) fibration.

For $X^{\prime} \subset X$, the map $p: X \rightarrow B$ is a (strong) relative fibration if there are path lifting function and shift $\Lambda^{p}$ and $\sigma^{p}$ such that

$$
\Lambda^{p} \mid W^{p \mid X^{\prime}} \quad \text { and } \quad \sigma^{p} \mid W^{p \mid X^{\prime}}
$$

are path lifting function and shift for $p \mid X^{\prime}$. With the added hypothesis that $p$ is a strong right $H$-fibration and

$$
a \mid X^{\prime} \times H: X^{\prime} \times H \rightarrow X^{\prime},
$$

we have the notion of a (strong) relative right $\mathrm{H}$-fibration.

The condition that a map $p: X \rightarrow B$ be a strong fibration is equivalent to the condition that the diagram

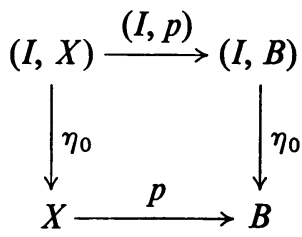

be a "weak pull-back," where $I$ is the interval $[0,1]$; i.e., if $f: Z \rightarrow(I, B)$ and $g: Z \rightarrow X$ are maps such that $\eta_{0} f=p g$, then there exists a map $h: Z \rightarrow(I, X)$, not necessarily unique, such that $(I, p) h=f$ and $\eta_{0} h=g$. The condition that $p$ be a strong right $H$-fibration is equivalent to (1.5) being a weak pull-back in the category $Q H$.

Dually, a morphism $i: A \rightarrow X$ is a cofibration provided the diagram

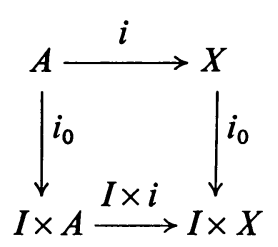

is a weak pushout, where $i_{0}(a)=(0, a)$, etc. This is equivalent to $i$ being an injection and $I \times A \cup 0 \times X$ being a retract of $I \times X$. The requirement that (1.6) be a weak pushout in the category $Q H$ defines a right $H$-cofibration.

We shall use the results that a composition of cofibrations is a cofibration and that a product of cofibrations is a cofibration; by the latter we mean that if $A \rightarrow X$ and $B \rightarrow Y$ are cofibrations, then so is $A \times Y \cup X \times B \rightarrow X \times Y$. The same conclusions hold for right $H$-cofibrations. 
Finally, we will use the following

Proposition (1.7). Let $p: X \rightarrow B$ be a (strong) left $H$-fibration and $\left(F, F^{\prime}\right)$ be a $Q H$-pair. Then

$$
\left(F \times{ }_{H} X, F^{\prime} \times_{H} X, \hat{p}, B\right)
$$

is a (strong) relative fibration. If $F^{\prime} \rightarrow F$ is a right $H$-cofibration, then $F^{\prime} \times{ }_{H} X \rightarrow$ $F \times{ }_{H} X$ is a cofibration.

(In the above statement the map $\hat{p}$ is defined by $p$ on the second factor.)

Since the category $Q$ is closed under disjoint unions and projections, it has arbitrary pushouts. That is, if

$$
A \stackrel{f_{\sigma}}{\longrightarrow} X_{\sigma}, \quad \sigma \in \Sigma,
$$

is a collection of maps, there exist a space $P$ and a collection $g_{\sigma}$ of maps

$$
X_{\sigma} \stackrel{g_{\sigma}}{\longrightarrow} P, \quad \sigma \in \Sigma,
$$

such that $g_{\sigma} f_{\sigma}: A \rightarrow P$ is independent of $\sigma$ and if

$$
X_{\sigma} \stackrel{h_{\sigma}}{\longrightarrow} R, \quad \sigma \in \Sigma,
$$

is a collection of maps such that $h_{\sigma} f_{\sigma}$ is independent of $\sigma$, there is a unique map $k: P \rightarrow R$ such that

$$
h_{\sigma}=k g_{\sigma} \text { for all } \sigma \in \Sigma .
$$

Definition (1.7). The category $Q^{2}$ is the category of pairs of spaces of $Q$ and maps of pairs. The category $P$ is the full subcategory of $Q^{2}$ in which the objects are pairs $(X, A)$ with the inclusion map $A \rightarrow X$ a cofibration. The category $Q^{\circ}$ is the full subcategory of $P$ in which the subspaces $A$ contain only one point. Finally, the category $P^{\circ}$ is the full subcategory of the category of triples $(X, A, C)$ and maps of triples in which each of the maps $A \rightarrow X$ and $C \rightarrow A$ are cofibrations and the space $C$ contains only one point.

A $Q H$-pair is a pair $\left(F, F^{\prime}\right)$ in $Q^{2}$ with the inclusion map $F^{\prime} \rightarrow F$ a $Q H$-morphism. The category $(Q H)^{2}$ is the category of $Q H$-pairs and $Q H$-maps of such pairs. The category $P H$ is the full subcategory of $(Q H)^{2}$ in which the objects have inclusion maps $F^{\prime} \rightarrow F Q H$-cofibrations. Then $(Q H)^{\circ}$ and $(P H)^{\circ}$. are defined similarly. For left $H$-spaces we have analogous categories $(H Q)^{2}, H P,(H Q)^{\circ}$, and $(H P)^{\circ}$.

II. Spectra. In the study of extraordinary homology theories, ring and module spectra play the role of coefficient rings and modules. In this section we review some of the basic definitions and develop some constructions which are used in $\S I I I$. A basic reference for this section is [14]. 
A spectrum $\mathscr{M}$ is a sequence of pairs $\left(M_{i}, \mu_{i}\right)$ of spaces $M_{i}$ and morphisms

$$
\mu_{i}: S^{1} \wedge M_{i} \rightarrow M_{i+1}
$$

in $Q^{\circ}, i=0,1,2, \ldots$

EXAMPLE (2.1). For a spectrum $\mathscr{M}$ and integer $n \in Z^{+}$, we denote by $\mathscr{M}(n)$ the spectrum $\mathscr{N}$ for which

$$
\begin{array}{cc}
N_{i}=* & \text { if } i<n, \\
N_{n+j}=M_{j} & \text { if } 0 \leqq j
\end{array}
$$

and

$$
\nu_{n+j}=\mu_{j} \quad\left(f^{n} \wedge M_{j}\right)
$$

if $0 \leqq j$, where $f: S^{1} \rightarrow S^{1}$ is the map of degree $(-1)$ given by $f\left(e^{i \theta}\right)=e^{-i \theta}$.

A morphism of spectra $f: \mathscr{M} \rightarrow \mathscr{N}$ is a sequence of morphisms $f_{i}: M_{i} \rightarrow N_{i}$ in $Q^{\circ}$ such that each of the diagrams

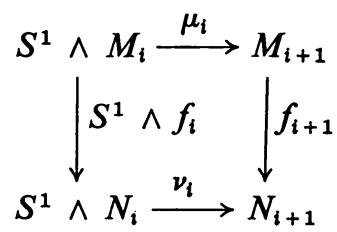

homotopy commutes. If the diagrams commute exactly, $f$ is called a strict morphism of spectra.

For a spectrum $\mathscr{M}$ the groups $\pi_{*}(\mathscr{M})$ are defined by

$$
\pi_{p}(\mathscr{M})=\lim \pi_{p+n}\left(M_{n}\right)
$$

(See pp. 243-244 of [14].) A morphism of spectra $f: \mathscr{M} \rightarrow \mathscr{N}$ induces homomorphisms $f_{*}: \pi_{p}(\mathscr{N}) \rightarrow \pi_{p}(\mathscr{N})$ by means of the homomorphisms

$$
f_{n_{\bullet}}: \pi_{p+n}\left(M_{n}\right) \rightarrow \pi_{p+n}\left(N_{n}\right) .
$$

For a space $X$ in $Q^{\circ}$ and a spectrum $\mathscr{M}$, a spectrum $\mathscr{N}=\mathscr{M} \wedge X$ is defined by

$$
N_{i}=M_{i} \wedge X \text { and } \nu_{i}=\mu_{i} \wedge X .
$$

The reduced (extraordinary) homology groups $\tilde{h}_{*}(X ; \mathscr{M})$ of $X$ with coefficients in the spectrum $\mathscr{M}$ are defined by

$$
\tilde{h}_{p}(X ; \mathscr{M})=\pi_{p}(\mathscr{M} \wedge X) .
$$

For $Y$ a space, the nonreduced groups $h_{*}(Y ; \mathscr{K})$ can be defined by

$$
h_{p}(Y ; \mathscr{M})=\tilde{h}_{p}\left(Y^{+} ; \mathscr{M}\right) .
$$

We note that Theorem 5.2 of [14] applies in $Q^{\circ}$; i.e., $\tilde{h}_{*}(; \mathscr{M})$ defines an extraordinary homology theory on the category $Q^{\circ},[7]$. 
Let $H$ denote a monoid. The spectrum $\mathscr{M}$ is a left $H$-spectrum if for each $i$, $M_{i}$ and $\mu_{i}$ are space and morphism in $(H Q)^{\circ}$, respectively. A right $H$-spectrum is defined similarly in terms of $(Q H)^{\circ}$.

A morphism of left (right) $H$-spectra is a morphism $f: \mathscr{M} \rightarrow \mathscr{N}$ of the left (right) $H$-spectra $\mathscr{M}$ and $\mathscr{N}$ such that each $f_{i}$ is a $(H Q)^{\circ}$ (or $\left.(Q H)^{\circ}\right)$ morphism and each of the diagrams (2.2) homotopy commutes in the category $(H Q)^{\circ}$ (or $\left.(Q H)^{\circ}\right)$. If each of the diagrams (2.2) commutes exactly, then $f$ is a strict morphism of left (right) $H$-spectra.

EXAMPLE (2.3). Let $\mathscr{M}$ be a spectrum with $H$ acting trivially on the spaces $M_{i}$ and let $X$ be a space in $(Q H)^{\circ}$. Then $\mathscr{N}=\mathscr{M} \wedge X$ is a right $H$-spectrum. If $f: \mathscr{M} \rightarrow \mathscr{M}^{\prime}$ is a (strict) morphism of spectra and $g: X \rightarrow X^{\prime}$ is a morphism of $(Q H)^{\circ}$, then

$$
f \wedge g: \mathscr{M} \wedge X \rightarrow \mathscr{M}^{\prime} \wedge X^{\prime}
$$

is a (strict) morphism of right $H$-spectra.

In [14], pairings of spectra were defined as the analogue of pairings of coefficient groups. We recall the definition.

DEFINITION (2.4). A pairing of spectra $t:(\mathscr{A}, \mathscr{B}) \rightarrow \mathscr{C}$ is a bisequence of morphisms of $Q^{\circ}$

$$
t_{p, q}: A_{p} \wedge B_{q} \rightarrow C_{p+q}
$$

such that

$$
t_{p+1, q}\left(\alpha_{p} \wedge B_{q}\right) \simeq \gamma_{p+q}\left(S^{1} \wedge t_{p, q}\right)
$$

and

$$
t_{p+1, q}\left(\alpha_{p} \wedge B_{q}\right) \simeq t_{p, q+1}\left(A_{p} \wedge \beta_{q}\right)\left(A_{p} \wedge f^{p} \wedge B_{q}\right)\left(\tau \wedge B_{q}\right)
$$

where $\tau: S^{1} \wedge A_{p} \rightarrow A_{p} \wedge S^{1}$ is the switching map and $f$ is the map of degree $(-1)$ given by $f\left(e^{i \theta}\right)=e^{-i \theta}$.

If $\alpha \in \pi_{p+m}\left(A_{p}\right)$ and $\beta \in \pi_{q+n}\left(B_{q}\right)$, then $(\alpha, \beta) \rightarrow t_{p, q_{*}}(\alpha \wedge \beta)$ induces a pairing $t_{p, q}^{\prime}: \pi_{p+m}\left(A_{p}\right) \otimes \pi_{q+n}\left(B_{q}\right) \rightarrow \pi_{p+q+m+n}\left(C_{p+q}\right)$. The homomorphisms $(-1)^{m a} t_{p, q}^{\prime}$ define a pairing

$$
\boldsymbol{t}_{*}: \pi_{m}(\mathscr{A}) \otimes \pi_{n}(\mathscr{B}) \rightarrow \pi_{m+n}(\mathscr{C}) .
$$

(See $[14$, p. 256], for details.)

EXAMPLES (2.5). (a) Let $\mathscr{S}$ be the sphere spectrum: $\left(S^{i}, \sigma_{i}\right)$, where

$$
\sigma_{i}: S^{1} \wedge S^{i} \stackrel{\cong}{\longrightarrow} S^{1+i}
$$

Let $\mathscr{A}$ be any spectrum and let

$$
\varepsilon_{i, j}: S^{i} \wedge A_{j} \rightarrow A_{i+j}
$$

be homotopic to the composition

$$
\left(S^{\circ} \wedge \alpha_{i+j-1}\right)\left(S^{1} \wedge \alpha_{i+j-1}\right) \cdots \cdots\left(S^{i-1} \wedge \alpha_{j}\right)
$$

for $i>0$ and $\varepsilon_{0, j}=A_{j}$. Then $\epsilon:(\mathscr{S}, \mathscr{A}) \rightarrow \mathscr{A}$ is a pairing [14, p. 255]. 
(b) Let $\varepsilon_{i, j}^{\prime}$ be homotopic to the composition.

$$
A_{i} \wedge S^{j} \stackrel{\tau}{\longrightarrow} S^{j} \wedge A_{i} \stackrel{\left(f_{j}\right)^{i} \wedge A_{i}}{\longrightarrow} S^{j} \wedge A_{i} \stackrel{\varepsilon_{j, i}}{\longrightarrow} A_{i+j},
$$
where $f_{j}=f \wedge f \wedge \cdots \wedge f: S^{j} \rightarrow S^{j}$. The maps $\varepsilon_{i, j}^{\prime}$ define a pairing $\boldsymbol{\epsilon}^{\prime}:(\mathscr{A}, \mathscr{S}) \rightarrow \mathscr{A}$.

In [14] G. W. Whitehead introduced the notion of a ring spectrum as the analogue of a coefficient ring in ordinary homology. We recall his definition and give a more restrictive definition: a strict ring spectrum, which is suited to later constructions in this and the next section

Definition (2.7). A (strict) ring spectrum is a triple $(\mathscr{R}, \boldsymbol{m}, \boldsymbol{u})$ in which $\mathscr{R}$ is a spectrum, $\boldsymbol{m}$ is a pairing $\boldsymbol{m}:(\mathscr{R}, \mathscr{R}) \rightarrow \mathscr{R}$, and $\boldsymbol{u}$ is a (strict) morphism $\boldsymbol{u}: \mathscr{S} \rightarrow \mathscr{R}$, where $\mathscr{S}$ is the sphere spectrum, such that

$$
\begin{aligned}
m_{i+j, k}\left(m_{i, j} \wedge R_{k}\right) & \simeq m_{i, j+k}\left(R_{i} \wedge m_{j, k}\right), \\
m_{i, j}\left(u_{i} \wedge R_{j}\right) & \simeq \varepsilon_{i, j} \quad\left(=R_{j} \text { if } i=0\right),
\end{aligned}
$$

and

$$
m_{i, j}\left(R_{i} \wedge u_{j}\right) \simeq \varepsilon_{i, j}^{\prime} \quad\left(=R_{i} \text { if } j=0\right)
$$

(all homotopies are replaced by equalities).

The morphism $\boldsymbol{u}$ is called the unit of the ring spectrum. The requirement that (1), (2) and (3) hold only up to a homotopy imply that the pairing

$$
m_{*}: \pi_{*}(\mathscr{R}) \otimes \pi_{*}(\mathscr{R}) \rightarrow \pi_{*}(\mathscr{R})
$$

makes $\pi_{*}(\mathscr{R})$ an associative, graded ring with unit represented by $u_{n}: S^{n} \rightarrow R_{n}$.

REMARK (2.8). If one is given a pairing $m:(\mathscr{R}, \mathscr{R}) \rightarrow \mathscr{R}$ and a morphism (not necessarily strict) $\boldsymbol{u}: \mathscr{S} \rightarrow \mathscr{R}$ which satisfy (1) exactly and (2) and (3) only up to homotopy, by letting $\hat{u}_{n}$ be the composition

$$
S^{1} \wedge \cdots \wedge S^{1} \stackrel{u_{1} \wedge \cdots \wedge u_{1}}{\longrightarrow} R_{1} \wedge \cdots \wedge R_{1} \longrightarrow R_{n},
$$

$\hat{\varepsilon}_{i, j}=m_{i, j}\left(\hat{u}_{i} \wedge R_{j}\right)$ and $\hat{\varepsilon}_{i, j}^{\prime}=m_{i, j}\left(R_{i} \wedge \hat{u}_{j}\right)$, we obtain a strict morphism $\hat{u}: \mathscr{S} \rightarrow \mathscr{R}$ and pairings $\hat{\varepsilon}:(\mathscr{S}, \mathscr{R}) \rightarrow \mathscr{R}$ and $\hat{\varepsilon}^{\prime}:(\mathscr{R}, \mathscr{S}) \rightarrow \mathscr{R}$ under which $(\mathscr{R}, \boldsymbol{m}, \hat{u})$ is a strict ring spectrum. The induced structure of graded ring with unit on $\pi_{*}(\mathscr{R})$ is unaltered.

REMARK. As was seen in (2.8), the equality (rather than homotopy) in conditions (2) and (3) of (2.7) is not a serious restriction and is imposed only as a technical convenience. The equality in condition (1) is a serious restriction, but something of this sort seems necessary to carry out the constructions of §III.

EXAMPLES (2.9). (a) The sphere spectrum $\mathscr{S}$ with

$$
m_{i, j}: S^{i} \wedge S^{j} \stackrel{\cong}{\longrightarrow} S^{i+j}
$$

and unit $\boldsymbol{u}=\mathscr{S}$ is a strict ring spectrum. This is the spectrum associated with stable homotopy as an extraordinary homology theory. The product in $\pi_{*}(\mathscr{S})$ corresponds to stable composition [3]. 
(b) For $\Lambda$ a ring with unit, let $R_{n}$ be the geometric realization in $Q^{\circ}$ [10] of the standard semisimplicial representation of $K(\Lambda, n)$, [8]. Then $\mathscr{R}$ is a strict ring spectrum.

(c) Let $(\mathscr{R}, \boldsymbol{m}, \boldsymbol{u})$ be a (strict) ring spectrum and $(H, \mu, e)$ be a topological monoid. The $H^{+} \wedge \mathscr{R}$ can be given a (strict) ring spectrum structure by letting

$$
\hat{m}_{i, j}=\left(\mu^{+} \wedge m_{i, j}\right)\left(H^{+} \wedge \tau \wedge R_{j}\right)
$$

and $\hat{u}_{p}$ be the composition

$$
S^{p} \longrightarrow S^{\circ} \wedge S^{p} \longrightarrow(e,+) \wedge S^{p} \longrightarrow H^{+} \wedge S^{p} \stackrel{H^{+} \wedge u_{p}}{\longrightarrow} H^{+} \wedge R_{p} .
$$

(d) The bordism theories associated with $O, U, \mathrm{Sp}, S O$ and $S U$ can be represented by strict ring spectra in the sense of (2.6), [9].

Definition (2.10). If $(\mathscr{R}, \boldsymbol{m}, \boldsymbol{u})$ is a (strict) ring spectrum, the spectrum $\mathscr{M}$ is a (strict) right $\mathscr{R}$-spectrum if there is a pairing $\gamma:(\mathscr{M}, \mathscr{R}) \rightarrow \mathscr{M}$ such that

$$
\begin{aligned}
\gamma_{i+j, k}\left(\gamma_{i, j} \wedge R_{k}\right) & \simeq \gamma_{i, j+k}\left(M_{i} \wedge m_{j, k}\right), \\
\gamma_{i, j}\left(M_{i} \wedge u_{j}\right) & \simeq \varepsilon_{i, j}^{\prime}
\end{aligned}
$$

and

$$
\mu_{i} \simeq \varepsilon_{i, 1}^{\prime}\left(M_{i} \wedge(f)^{i}\right) \tau,
$$

(all homotopies are replaced by equalities), where $\tau: S^{1} \wedge M_{i} \rightarrow M_{i} \wedge S^{1}$ is the switching map. A (strict) left $\mathscr{R}$-spectrum is defined similarly.

Comments similar to those of Remark (2.8) apply to the equality (rather than homotopy) in conditions (2) and (3).

It is easy to see that $\gamma_{*}$ makes $\pi_{*}(\mathscr{M})$ a module over $\pi_{*}(\mathscr{R})$.

DEFINITION (2.11). A morphism of (strict) right $\mathscr{R}$-spectra is a spectrum morphism $f: \mathscr{M} \rightarrow \mathscr{N}$ of (strict) right $\mathscr{R}$-spectra such that

$$
n_{i, j}\left(f_{i} \wedge R_{j}\right) \simeq f_{i+j} m_{i, j}
$$

( $\simeq$ replaced by $=$ ). A morphism of (strict) left $\mathscr{R}$-spectra is defined similarly.

REMARK (2.12). Condition (3) of Definition (2.10) implies that a morphism of strict right $\mathscr{R}$-spectra is a strict morphism of spectra.

Clearly, for a morphism $f: \mathscr{M} \rightarrow \mathscr{N}$ of right $\mathscr{R}$-spectra, the induced morphism $f_{*}: \pi_{*}(\mathscr{M}) \rightarrow \pi_{*}(\mathscr{N})$ is a right $\pi_{*}(\mathscr{R})$-module homomorphism.

EXAMPLES (2.13). (a) Every spectrum can be made into a strict left or right $\mathscr{S}$-spectrum.

(b) If $\mathscr{R}$ is a strict ring spectrum, then (changing if necessary the maps $\rho_{i}$ by a homotopy to satisfy condition (3) of Definition (2.10)) $\mathscr{R}$ is a strict right $\mathscr{R}$ spectrum.

(c) If $X$ is an object of $Q^{\circ}$ and $\mathscr{M}$ is a (strict) right [left] $\mathscr{R}$-spectrum, then, using the obvious pairings, $\mathscr{M} \wedge X$ is a (strict) right [left] $\mathscr{R}$-spectrum. In particular, $\mathscr{R} \wedge X$ is a strict right $\mathscr{R}$-spectrum if $\mathscr{R}$ is a strict ring spectrum. 
(d) If $Y$ is an object of $(Q H)^{\circ}, \mathscr{R}$ is a (strict) ring spectrum and $\mathscr{M}$ is a (strict) right $\mathscr{R}$-spectrum, then $\mathscr{M} \wedge Y$ is given the structure of a (strict) right $\mathscr{R} \wedge H^{+}$spectrum by the composition

$$
\left(M_{i} \wedge Y\right) \wedge\left(R_{j} \wedge H^{+}\right) \rightarrow\left(M_{i} \wedge R_{j}\right) \wedge\left(Y \wedge H^{+}\right) \rightarrow M_{i+j} \wedge Y .
$$

Thus, $\pi_{*}(\mathscr{M} \wedge Y)$ is a right $\pi_{*}\left(\mathscr{R} \wedge H^{+}\right)$-module; equivalently, $\tilde{h}_{*}(Y ; \mathscr{M})$ is a right module over $h_{*}(H ; \mathscr{R})$.

(e) With the monoid $H$ acting trivially on the left factor, the space $S^{i} \wedge H^{+}$ $\equiv S^{i} \times H /_{*} \times H$ is an object of $(Q H)^{\circ}$. Also, $\tilde{h}_{*}\left(S^{i} \wedge H^{+} ; \mathscr{R}\right)=\pi_{*}\left(\mathscr{R} \wedge\left(S^{i} \wedge H^{+}\right)\right)$. Thus, $\tilde{h}_{*}\left(S^{i} \wedge H^{+} ; \mathscr{R}\right)$ is a free right $h_{*}(H ; \mathscr{R})$-module on one generator; this generator is in dimension $i$ and is represented by the composition

$$
S^{n+i} \cong S^{n} \wedge S^{i} \wedge S^{\circ} \rightarrow S^{n} \wedge S^{i} \wedge(e,+) \rightarrow R_{n} \wedge S^{i} \wedge H^{+},
$$

(see $[14,(5.1)])$.

(f) If $\mathscr{M}$ is a strict left (right) $\mathscr{R}$-spectrum and $X$ is an object of $Q^{\circ}$, then $(X, \mathscr{M})$ is a strict left (right) $\mathscr{R}$-spectrum.

Proof of Example (2.13)(f). The spectrum $(X, \mathscr{M})$ has spaces $(X, \mathscr{M})_{i}=\left(X, M_{i}\right)^{\circ}$ and maps

$$
\pi_{i}: S^{1} \wedge(X, \mathscr{M})_{i} \rightarrow(X, \mathscr{M})_{i+1}
$$

the compositions

$$
S^{1} \wedge\left(X, M_{i}\right)^{\circ} \rightarrow\left(X, S^{1} \wedge M_{i}\right)^{\circ} \rightarrow\left(X, M_{i+1}\right)^{\circ},
$$

in which the first map is adjoint to

$$
\left(X, M_{i}\right)^{\circ} \rightarrow\left(S^{1} \wedge X, S^{1} \wedge M_{i}\right)^{\circ} \cong\left(S^{1},\left(X, S^{1} \wedge M_{i}\right)^{\circ}\right)^{\circ}
$$

and the second is $\left(X, \mu_{i}\right)^{\circ}$. The base point of $\left(X, M_{i}\right)^{\circ}$ is $(X, *)$ and it can be verified the inclusion map of this base point is a cofibration. We can write a formula for $\pi_{i}$ as follows:

$$
\pi_{i}(s, f)(x)=\mu_{i}(s \wedge f x) .
$$

The proof of the lemma consists of a number of routine verifications. Consider the left case.

$$
\bar{\gamma}: \mathscr{R} \wedge(X, \mathscr{M}) \rightarrow(X, \mathscr{M})
$$

is given by the compositions $\bar{\gamma}_{i, j}$

$$
R_{i} \wedge\left(X, M_{j}\right)^{\circ} \rightarrow\left(X, R_{i} \wedge M_{j}\right)^{\circ} \rightarrow\left(X, M_{i+j}\right)^{\circ}
$$

or equally the formula

$$
\bar{\gamma}_{i, j}(r, f)(x)=\gamma_{i, j}(r \wedge f x) .
$$

We check the equality

$$
\bar{\gamma}_{i+j_{, k}} \cdot\left(m_{i, j} \wedge\left(X, M_{k}\right)^{\circ}\right)=\bar{\gamma}_{i, j+k} \cdot R_{i} \wedge \bar{\gamma}_{j, k} .
$$


Let $s \in R_{i}, t \in R_{j}, x \in M_{k}$ and $f \in\left(X, M_{k}\right)^{\circ}$. Then

$$
\begin{aligned}
\bar{\gamma}_{i+j, k}\left(m_{i, j} \wedge\left(X, M_{k}\right)^{\circ}\right)(s \wedge t \wedge f)(x) & =\bar{\gamma}_{i+j, k}\left(m_{i, j}(s \wedge t) \wedge f\right)(x) \\
& =\gamma_{i+j, k}\left(m_{i, j}(s \wedge t) \wedge f x\right) \\
& =\gamma_{i, j+k}\left(s \wedge \gamma_{j, k}(t \wedge f x)\right) \\
& =\gamma_{i, j+k}\left(s \wedge \bar{\gamma}_{j, k}(t, f)(x)\right) \\
& =\bar{\gamma}_{i, j+k}\left(s, \bar{\gamma}_{j, k}(t, f)\right)(x) \\
& =\bar{\gamma}_{i, j+k} \cdot R_{i} \wedge \bar{\gamma}_{j, k}(s, t, f)(x) .
\end{aligned}
$$

The other equalities are verified in similar ways.

Definition (2.14). A cofibration of spectra is a strict morphism $i: \mathscr{A} \rightarrow \mathscr{X}$ of spectra such that for each $n, i_{n}: A_{n} \rightarrow X_{n}$ is a cofibration. If $i: \mathscr{X}$ is a cofibration of spectra, then $(\mathscr{X}, \mathscr{A})$ is called a pair of spectra.

If $(\mathscr{X}, \mathscr{A})$ is a pair of spectra, the groups $\pi_{*}(\mathscr{X}, \mathscr{A})$ are defined by

$$
\pi_{p}(\mathscr{X}, \mathscr{A})=\lim _{\rightarrow} \pi_{p+n}\left(X_{n}, A_{n}\right) \text {. }
$$

If $(\mathscr{X}, \mathscr{A})$ and $(\mathscr{Y}, \mathscr{B})$ are pairs of spectra, a strict morphism $f:(\mathscr{X}, \mathscr{A}) \rightarrow(\mathscr{Y}, \mathscr{B})$ is a strict morphism $f: \mathscr{X} \rightarrow \mathscr{Y}$ such that for each $n, f_{n} \mid A_{n}: A_{n} \rightarrow B_{n}$. In this case, $f$ induces

$$
f_{*}: \pi_{*}(\mathscr{X}, \mathscr{A}) \rightarrow \pi_{*}(\mathscr{Y}, \mathscr{B})
$$

Since the diagrams

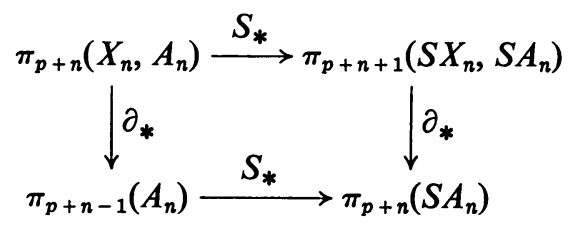

anticommute, the homomorphisms

$$
(-1)^{n} \partial_{*}: \pi_{p+n}\left(X_{n}, A_{n}\right) \rightarrow \pi_{p-1+n}\left(A_{n}\right)
$$

define a homomorphism $\partial_{*}: \pi_{p}(\mathscr{X}, \mathscr{A}) \rightarrow \pi_{p-1}(\mathscr{A})$. As the direct limit of exact sequences is exact, the sequence

$$
\cdots \rightarrow \pi_{p}(\mathscr{A}) \rightarrow \pi_{p}(\mathscr{X}) \rightarrow \pi_{p}(\mathscr{X}, \mathscr{A}) \rightarrow \pi_{p-1}(\mathscr{A}) \rightarrow \cdots
$$

is exact. The exact sequence (2.15) is called the homotopy exact sequence of the pair $(\mathscr{X}, \mathscr{A})$, and it is natural with respect to strict morphisms of pairs of spectra and their induced homomorphisms.

If $i: \mathscr{A} \rightarrow \mathscr{X}$ is a cofibration of spectra, then $* \rightarrow \mathscr{X} / \mathscr{A}$ is a cofibration of spectra and the morphism $(\mathscr{X}, \mathscr{A}) \rightarrow(\mathscr{X} / \mathscr{A}, *)$ is a strict morphism of pairs.

Proposition (2.16). If $i: \mathscr{A} \rightarrow \mathscr{X}$ is a cofibration of spectra, then

$$
\pi_{*}(\mathscr{X}, \mathscr{A}) \stackrel{\cong}{\longrightarrow} \pi_{*}(\mathscr{X} / \mathscr{A}, *)
$$


Proof. This is an obvious double limit argument using the isomorphism

$$
\pi_{*}(X, A) \stackrel{\cong}{\longrightarrow} \pi_{*}(X \cup T A, T A)
$$

in the stable range.

Proposition (2.17). Let $\Sigma$ be an index set, $\Sigma^{\prime}=\Sigma \cup\{0\}, \mathscr{A}, \mathscr{X}^{\sigma}, \sigma \in \Sigma^{\prime}$, be spectra, and

$$
f^{\sigma}: \mathscr{A} \rightarrow \mathscr{X}^{\sigma}, \quad \sigma \in \Sigma^{\prime},
$$

be a collection of strict morphisms of spectra such that each $f^{\sigma}$ is a cofibration for $\sigma \in \Sigma$. Then there exist a spectrum $\mathscr{P}$ and a collection

$$
\boldsymbol{g}^{\sigma}: \mathscr{X}^{\sigma} \rightarrow \mathscr{P}, \quad \sigma \in \Sigma^{\prime},
$$

of strict morphisms of spectra such that the diagram

$$
\mathscr{A} \stackrel{f^{\tau}}{\longrightarrow} \mathscr{X}^{\tau} \stackrel{g^{\tau}}{\longrightarrow} \mathscr{P}, \quad \tau \in \Sigma^{\prime}
$$

is a push-out is the category of spectra and strict morphisms of spectra. Furthermore, $\mathrm{g}^{\circ}$ is a cofibration of spectra.

Proof. For each $i$ there exist a space $P_{i}$ and morphisms

$$
g_{i}^{\sigma}: X_{i}^{\sigma} \rightarrow P_{i}
$$

such that

$$
A_{i} \stackrel{f_{i}^{\tau}}{\longrightarrow} X_{i}^{\tau} \stackrel{g_{i}^{\tau}}{\longrightarrow} P_{i}
$$

is a pushout in $Q$. Let $p_{i}=g_{i}^{\circ} f_{i}^{\circ} a_{i}$, where $a_{i}$ is the base point of $A_{i}$. We shall first show that $g_{i}^{\circ}$ is a cofibration. Since $x_{i}^{\circ} \rightarrow X_{i}^{\circ}$ is a cofibration, and the composition of cofibration is a cofibration, it follows that $p_{i} \rightarrow P_{i}$ is a cofibration and the $g_{i}^{\tau}$ are $Q^{\circ}$-morphisms.

Consider the diagram

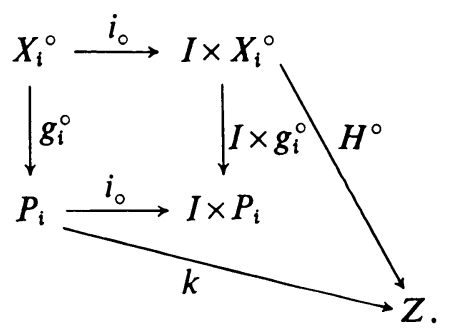

We seek a map $K: I \times P_{i} \rightarrow Z$ such that $K i_{\circ}=k$ and $K \circ\left(I \times g_{i}^{\circ}\right)=H^{\circ}$. 
For each $\sigma \in \Sigma$ we have the commutative diagram

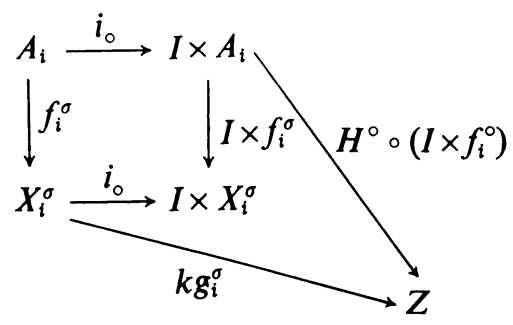

and the resultant map $H^{\sigma}: I \times X_{i}^{\sigma} \rightarrow Z$ such that $H^{\sigma} i_{\circ}=k g_{i}^{\sigma}$ and $H^{\sigma} \circ\left(I \times f_{i}^{\sigma}\right)$ $=H^{\circ} \circ\left(I \times f_{i}^{\circ}\right)$, recalling that $f_{i}^{\sigma}$ is a cofibration.

Since (2.18) is a pushout in $Q$, so is

$$
I \times A_{i} \stackrel{I \times f_{i}^{\tau}}{\longrightarrow} I \times X_{i}^{\tau} \stackrel{I \times g_{i}^{\tau}}{\longrightarrow} I \times P_{i} .
$$

But we have a collection of maps

$$
H^{\tau}: I \times X_{i}^{\tau} \rightarrow Z, \quad \tau \in \Sigma^{\prime},
$$

such that $H^{\tau} \circ\left(I \times f_{i}^{\tau}\right)$ is independent of $\tau$.

Thus, there is a map $K: I \times P_{i} \rightarrow Z$ such that

$$
K \circ\left(I \times g_{i}^{\tau}\right)=H^{\tau}, \quad \tau \in \Sigma^{\prime} .
$$

In particular,

$$
K \circ\left(I \times g_{i}^{\circ}\right)=H^{\circ} .
$$

Also,

$$
K^{\circ} i_{\circ} \circ g_{i}^{\tau}=K^{\circ}\left(I \times g_{i}^{\tau}\right) \circ i_{\circ}=H^{\tau} i_{\circ}=k g_{i}^{\tau}
$$

for $\tau \in \Sigma^{\prime}$. But $k g_{i}^{\tau}: X_{i}^{\tau} \rightarrow Z$ is a family of maps such that

$$
k g_{i}^{\tau} f_{i}^{\tau}=H^{\circ} \circ\left(I \times f_{i}^{\circ}\right) i_{\circ}
$$

is independent of $\tau$. There is then a unique map

$$
l: P_{i} \rightarrow Z
$$

such that $l g_{i}^{\tau}=k g_{i}^{\tau}$ for all $\tau \in \Sigma^{\prime}$. But $k$ and $K \circ i_{\circ}$ are clearly such maps. They are then the same map.

Thus, (2.19) is a weak pushout and $g_{i}^{\circ}$ is a cofibration for each $i$.

Since (2.18) is a pushout, so is

$$
S^{1} \wedge A_{i} \stackrel{S^{1} \wedge f_{i}^{\tau}}{\longrightarrow} S^{1} \wedge X_{i}^{\tau} \stackrel{S^{1} \wedge g_{i}^{\tau}}{\longrightarrow} S^{1} \wedge P_{i} .
$$


The diagrams

$$
\begin{aligned}
& S^{1} \wedge A_{i} \stackrel{\alpha_{i}}{\longrightarrow} A_{i+1} \\
& \begin{array}{l}
\downarrow S^{1} \wedge f_{i}^{\tau} \\
S^{1} \wedge X_{i}^{\tau} \stackrel{\varepsilon_{i}^{\tau}}{\longrightarrow} X_{i+1}^{\tau}
\end{array}
\end{aligned}
$$

commute, and so, the family

$$
g_{i+1}^{\tau} \varepsilon_{i}^{\tau}: S^{1} \wedge X_{i}^{\tau} \rightarrow P_{i+1}
$$

is such that

$$
g_{i+1}^{\tau} \varepsilon_{i}^{\tau} S^{1} \wedge f_{i}^{\tau}=g_{i+1}^{\tau} f_{i+1}^{\tau} \alpha_{i} .
$$

But $g_{i+1}^{\tau} f_{i+1}^{\tau}$ is independent of $\tau$. Thus, $g_{i+1}^{\tau} \varepsilon_{i}^{\tau} S^{1} \wedge f_{i}^{\tau}$ is independent of $\tau$ and there is a unique morphism

$$
\pi_{i}: S^{1} \wedge P_{i} \rightarrow P_{i+1}
$$

in $Q^{\circ}$ such that

$$
\pi_{i} S^{1} \wedge f_{i}^{\tau}=g_{i+1}^{\tau} \varepsilon_{i}^{\tau}, \quad \tau \in \Sigma^{\prime} .
$$

Thus, for the spectrum $\mathscr{P}=\left(P_{i}, \pi_{i}\right)$, we have

$$
\boldsymbol{g}^{\tau}: \mathscr{X}^{\tau} \rightarrow \mathscr{P}, \quad \tau \in \Sigma^{\prime},
$$

are strict morphisms and $g^{\circ}$ is a cofibration.

Finally, to see that

$$
\mathscr{A} \stackrel{f^{\tau}}{\longrightarrow} \mathscr{X}^{\imath} \stackrel{g^{\tau}}{\longrightarrow} \mathscr{P}, \quad \tau \in \Sigma^{\prime},
$$

is a pushout, let

$$
\boldsymbol{h}^{\tau}: \mathscr{X}^{\tau} \rightarrow \mathscr{B}, \quad \tau \in \Sigma^{\prime},
$$

be a family of strict morphisms such that $\boldsymbol{h}^{\tau} \boldsymbol{f}^{\imath}$ is independent of $\tau$. For each $i$, there is a unique morphism

$$
k_{i}: P_{i} \rightarrow B_{i}
$$

in $Q^{\circ}$ such that

$$
k_{i} g_{i}^{\tau}=h_{i}^{\tau} \quad \text { for } \tau \in \Sigma^{\prime} .
$$

We have only to verify that

$$
\boldsymbol{k}: \mathscr{P} \rightarrow \mathscr{B}
$$

is a strict morphism of spectra. But this follows as before from the facts that the $h^{z}$ are strict morphisms and that for each $i$ the diagram (2.20) is a pushout. 
Definition $(2.21)$. Let $(\mathscr{X}, \mathscr{A}),(\mathscr{Y}, \mathscr{B}),(\mathscr{Z}, \mathscr{C})$, and $(\mathscr{C}, \mathscr{D})$ be pairs of spectra. $A$ relative pairing

$$
t:((\mathscr{X}, \mathscr{A}),(\mathscr{Y}, \mathscr{B})) \rightarrow(\mathscr{Z}, \mathscr{B}, \mathscr{D})
$$

is a pairing $t:(\mathscr{X}, \mathscr{Y}) \rightarrow \mathscr{Z}$ such that restrictions of the maps and homotopies which make $t$ a pairing define pairings $(\mathscr{X}, \mathscr{B}) \rightarrow \mathscr{C},(\mathscr{A}, \mathscr{Y}) \rightarrow \mathscr{C}$ and $(\mathscr{A}, \mathscr{B}) \rightarrow \mathscr{D}$.

It is not difficult to see that a relative pairing induces a pairing

$$
(\mathscr{X} / \mathscr{A}, \mathscr{Y} / \mathscr{B}) \rightarrow \mathscr{Z} / \mathscr{C} \text {. }
$$

Let $t$ be a pairing as in (2.21). For $\alpha \in \pi_{p+m}\left(X_{p}, A_{p}\right)$ and $\beta \in \pi_{q+n}\left(Y_{q}, B_{q}\right)$,

$$
(\alpha, \beta) \rightarrow t_{p, q .} \quad(\alpha \wedge \beta)
$$

determines a pairing

$$
t_{p, q}^{\prime}: \pi_{p+m}\left(X_{p}, A_{p}\right) \otimes \pi_{q+n}\left(Y_{q}, B_{q}\right) \rightarrow \pi_{p+q+m+n}\left(Z_{p+q}, C_{p+q}\right) .
$$

The homomorphisms $(-1)^{m q} t_{p, q}^{\prime}$ then define a pairing

$$
\boldsymbol{t}_{*}: \pi_{m}(\mathscr{X}, \mathscr{A}) \otimes \pi_{n}(\mathscr{Y}, \mathscr{B}) \rightarrow \pi_{m+n}(\mathscr{Z}, \mathscr{C}) .
$$

Definition (2.22). For $\mathscr{R}$ a (strict) ring spectrum, the pair $(\mathscr{X}, \mathscr{A})$ of spectra is a (strict) right $\mathscr{R}$-pair of spectra if $\mathscr{X}$ is a (strict) right $\mathscr{R}$-spectrum and the pairing $\gamma:(\mathscr{X}, \mathscr{R}) \rightarrow \mathscr{X}$ defines a relative pairing

$$
\gamma:((\mathscr{X}, \mathscr{A}),(\mathscr{R}, *)) \rightarrow(\mathscr{X}, \mathscr{A}, *) \text {. }
$$

A right $\mathscr{R}$-morphism of right $\mathscr{R}$-pairs is a (strict) morphism $f:(\mathscr{X}, \mathscr{A}) \rightarrow(\mathscr{Y}, \mathscr{B})$ of pairs such that $f: \mathscr{X} \rightarrow \mathscr{Y}$ is a morphism of (strict) right $\mathscr{R}$-spectra.

The homotopy morphism induced by a right $\mathscr{R}$-morphism of pairs is a right $\pi_{*}(\mathscr{R})$-module homomorphism.

Proposition (2.23). Let $\Sigma$ be an index set, $\Sigma^{\prime}=\Sigma \cup\{0\}, \mathscr{R}$ be a strict ring spectrum, and

$$
f^{\sigma}: \mathscr{A} \rightarrow \mathscr{X}^{\sigma}, \quad \sigma \in \Sigma^{\prime}
$$

be a collection of morphisms of strict left $\mathscr{R}$-spectra such that for each $\sigma \in \Sigma, f^{\sigma}$ is a cofibration.

Then there exist a strict left $\mathscr{R}$-spectrum $\mathscr{P}$ and a collection

$$
\boldsymbol{g}^{\sigma}: \mathscr{X}^{\sigma} \rightarrow \mathscr{P}, \quad \sigma \in \Sigma^{\prime},
$$

of morphisms of strict left $\mathscr{R}$-spectra such that the diagram

$$
\mathscr{A} \stackrel{f^{\sigma}}{\longrightarrow} \mathscr{X}^{\sigma} \stackrel{g^{\sigma}}{\longrightarrow} \mathscr{P}, \quad \sigma \in \Sigma^{\prime},
$$

is a pushout in the category of strict left $\mathscr{R}$-spectra and their morphisms. Furthermore, $\left(\mathscr{P}, \mathscr{X}^{\circ}\right)$ is a strict left $\mathscr{R}$-pair of spectra. 
Proof. We shall verify that for the $\mathscr{P}$ and $\boldsymbol{g}^{\sigma}$ of Proposition (2.17),

(a) $\mathscr{P}$ is a strict left $\mathscr{R}$-spectrum,

(b) each $\boldsymbol{g}^{\sigma}$ is a morphism of strict left $\mathscr{R}$-spectra,

(c) if $\boldsymbol{k}^{\sigma}: \mathscr{X}^{\sigma} \rightarrow \mathscr{B}, \sigma \in \Sigma^{\prime}$, is a family of morphisms of strict left $\mathscr{R}$-spectra, the unique morphism $k: \mathscr{P} \rightarrow \mathscr{B}$ such that $\boldsymbol{k g}^{\sigma}=\boldsymbol{h}^{\sigma} . \sigma \in \Sigma^{\prime}$, given by Proposition (2.17) is a morphism of strict left $\mathscr{R}$-spectra.

Note the conclusion that $\left(\mathscr{P}, \mathscr{X}^{\circ}\right)$ is a strict left $\mathscr{R}$-pair of spectra is an immediate consequence of (b) for $\sigma=0$ and Proposition (2.17).

The pairing $\gamma:(\mathscr{R}, \mathscr{P}) \rightarrow \mathscr{P}$ is obtained from the pushout diagram

$$
R_{i} \wedge A_{j} \stackrel{R_{i} \wedge f_{j}^{\sigma}}{\longrightarrow} R_{i} \wedge X_{j}^{\sigma} \stackrel{R_{i} \wedge g_{j}^{\sigma}}{\longrightarrow} R_{i} \wedge P_{j}
$$

and the pairings

$$
R_{i} \wedge X_{j}^{\sigma} \stackrel{\lambda_{i, j}}{\longrightarrow} X_{i+j}^{\sigma} \stackrel{g_{i+j}^{\sigma}}{\longrightarrow} P_{i+j} .
$$

Since $f^{\sigma}$ are morphisms of strict left $\mathscr{R}$-spectra, the compositions

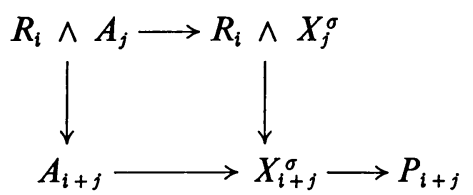

are independent of $\sigma$. Thus, there is a unique map

$$
\gamma_{i, j}: R_{i} \wedge P_{j} \rightarrow P_{i+j}
$$

such that

$$
\gamma_{i, j} \circ R_{i} \wedge g_{j}^{\sigma}=g_{i+j}^{\sigma} \lambda_{i, j} \text { for all } \sigma \in \Sigma^{\prime} .
$$

We claim this gives $\mathscr{P}$ the structure of a strict left $\mathscr{R}$-spectrum. Notice that the equations (2.24) are equivalent to assertion (b).

We verify first that the diagram

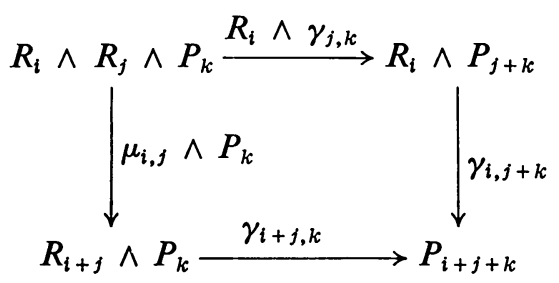

commutes. Since

$$
R_{i} \wedge R_{j} \wedge A_{k} \rightarrow R_{i} \wedge R_{j} \wedge X_{k}^{\sigma} \rightarrow R_{i} \wedge R_{j} \wedge P_{k}, \quad \sigma \in \Sigma^{\prime},
$$

is a pushout diagram in $Q$ and each of the compositions

$$
\begin{aligned}
g_{i+j+k}^{\sigma} \circ \lambda_{i+j, k}^{\sigma} \circ R_{i} \wedge \lambda_{j, k}^{\sigma} \circ R_{i} & \wedge R_{j} \wedge f_{k}^{\sigma} \\
& =g_{i+j+k}^{\sigma} \circ \lambda_{i+j, k}^{\sigma} \circ \mu_{i, j} \wedge X_{k}^{\sigma} \circ R_{i} \wedge R_{j} \wedge f_{k}^{\sigma}
\end{aligned}
$$


is independent of $\sigma$, there is a unique morphism

$$
t_{i, j, k}: R_{i} \wedge R_{j} \wedge P_{k} \rightarrow P_{i+j+k}
$$

such that

$$
t_{i, j, k} \circ R_{i} \wedge R_{j} \wedge g_{k}^{\sigma}=g_{i+j+k}^{\sigma} \circ \lambda_{i+j, k} \circ \mu_{i+j} \wedge X_{k}^{\sigma}, \quad \sigma \in \Sigma^{\prime} .
$$

But each of the morphisms

$$
\gamma_{i, j+k} \circ R_{i} \wedge \gamma_{j, k} \text { and } \gamma_{i+j, k} \circ \mu_{i, j} \wedge P_{k}
$$

has this property.

We next show that for $u: \mathscr{S} \rightarrow \mathscr{R}$ the unit of $\mathscr{R}$, that

$$
\varepsilon_{i, j}=\gamma_{i, j} \circ\left(u_{i} \wedge P_{j}\right) .
$$

For this we employ the pushout diagram

$$
S^{i} \wedge A_{j} \stackrel{S^{i} \wedge f_{j}^{\sigma}}{\longrightarrow} S^{i} \wedge X_{j}^{\sigma} \stackrel{S^{i} \wedge g_{j}^{\sigma}}{\longrightarrow} S^{i} \wedge P_{j} .
$$

The composition $\varepsilon_{i, j} \circ S^{i} \wedge g_{j}^{\sigma} \circ S^{i} \wedge A_{j}=g_{i+j}^{\sigma} \circ f_{i+j}^{\sigma} \circ \varepsilon_{i, j}$ is independent of $\sigma$. Thus, there is a unique morphism

such that

$$
v_{i, j}: S^{i} \wedge P_{j} \rightarrow P_{i+j}
$$

$$
v_{i, j} \circ S^{i} \wedge g_{j}^{\sigma}=\varepsilon_{i, j} \circ S^{i} \wedge g_{j}^{\sigma} \text { for all } \sigma \in \Sigma^{\prime} .
$$

But $\varepsilon_{i, j}$ and $\gamma_{i, j} \circ\left(u_{i} \wedge P_{j}\right)$ are such morphisms. The latter assertion follows via the equalities

The equality

$$
\begin{aligned}
\gamma_{i, j} \circ\left(u_{i} \wedge P_{j}\right) \circ\left(S^{i} \wedge g_{j}^{\sigma}\right) & =\gamma_{i, j} \circ R_{i} \wedge g_{j}^{\sigma} \circ u_{i} \wedge P_{j} \\
& =g_{i+j}^{\sigma} \circ \lambda_{i, j}^{\sigma} \circ u_{i} \wedge X_{j}^{\sigma} \\
& =g_{i+j}^{\sigma} \circ \varepsilon_{i, j}^{\sigma} \\
& =\varepsilon_{i, j} \circ\left(S^{i} \wedge g_{j}^{\sigma}\right) .
\end{aligned}
$$

$$
\gamma_{1, j} \circ u_{1} \wedge P_{j} \circ\left((f)^{j} \wedge P_{j}\right) \circ \tau=\varepsilon_{j, 1}^{\prime}
$$

follows similarly, using the pushout diagram

$$
A_{j} \wedge S^{1} \stackrel{f_{j}^{\sigma} \wedge S^{1}}{\longrightarrow} X_{j}^{\sigma} \wedge S^{1} \stackrel{g_{j}^{\sigma} \wedge S^{1}}{\longrightarrow} P_{j} \wedge S^{1}, \quad \sigma \in \Sigma^{\prime} .
$$

To complete the verification (a) we have to prove only that

$$
\gamma:(\mathscr{R}, \mathscr{P}) \rightarrow \mathscr{P}
$$

is indeed a pairing. That is, we need to show that

and

$$
\gamma_{p+1, q} \circ\left(\rho_{p} \wedge P_{q}\right) \simeq \pi_{p+q} \circ\left(S^{1} \wedge \gamma_{p, q}\right)
$$

$$
\gamma_{p+1, q} \circ\left(\rho_{p} \wedge P_{q}\right) \simeq \gamma_{p, q+1} \circ\left(R_{p} \wedge \pi_{\Omega}\right) \circ\left(R_{p} \wedge(f)^{p} \wedge P_{q}\right) \circ\left(\tau \wedge P_{q}\right)
$$


Actually in the presence of diagram (2.25) these two relations hold with homotopy replaced by equality.

The first equality follows from the commutative diagram

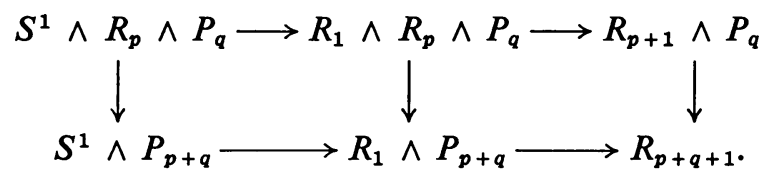

The second equality follows from the equations

$$
\begin{aligned}
\gamma_{p, q+1} \circ\left(R_{p}\right. & \left.\wedge \gamma_{1, q}\right) \circ\left(R_{p} \wedge u_{1} \wedge P_{q}\right) \circ\left(R_{p} \wedge(f)^{p} \wedge P_{q}\right) \circ\left(\tau \wedge P_{q}\right) \\
& =\gamma_{p+1, q} \circ\left(\mu_{p, 1} \wedge P_{q}\right) \circ\left(R_{p} \wedge u_{1} \wedge P_{q}\right) \circ\left(R_{p} \wedge(f)^{p} \wedge P_{q}\right) \circ\left(\tau \wedge P_{q}\right) \\
& =\gamma_{p+1, q} \circ\left(\mu_{p, 1} \circ\left(R_{p} \wedge u_{1}\right) \circ\left(R_{p} \wedge(f)^{p}\right) \circ \tau\right) \wedge P_{q} \\
& =\gamma_{p+1, q} \circ\left(\mu_{1, p} \circ\left(u_{1} \wedge R_{p}\right) \circ\left((f)^{p} \wedge R_{p}\right) \circ \tau \circ\left(R_{p} \wedge(f)^{p}\right) \circ \tau\right) \wedge P_{q} \\
& =\gamma_{p+1, q} \circ\left(\mu_{1, p} \circ\left(u_{1} \wedge R_{p}\right)\right) \wedge P_{q} \\
& =\gamma_{p+1, q} \circ \rho_{p} \wedge P_{q} .
\end{aligned}
$$

To verify (c) we show that for the morphism $k: \mathscr{P} \rightarrow \mathscr{B}$ of Proposition (2.17) obtained under our added hypothesis, the equalities

$$
\gamma_{p, q} \circ R_{p} \wedge k_{q}=k_{p+q} \circ \gamma_{p, q}
$$

hold. This follows immediately from the facts that

$$
R_{p} \wedge A_{q} \stackrel{R_{p} \wedge f_{q}^{\sigma}}{\longrightarrow} R_{p} \wedge X_{q}^{\sigma} \stackrel{R_{p} \wedge g_{q}^{\sigma}}{\longrightarrow} R_{p} \wedge P_{q}, \quad \sigma \in \Sigma^{\prime},
$$

is a pushout diagram, that the composition

$$
\gamma_{p, q} \circ R_{p} \wedge h_{q}^{\sigma} \circ R_{p} \wedge f_{q}^{\sigma}
$$

is independent of $\sigma$, and thus that the morphism $\omega_{p, q}: R_{p} \wedge P_{q} \rightarrow B_{p+q}$ such that

$$
\omega_{p, q} \circ R_{p} \wedge g_{q}^{\sigma}=\gamma_{p, q} \circ R_{p} \wedge h_{q}^{\sigma}, \quad \sigma \in \Sigma^{\prime},
$$

is unique. But each of the morphisms in (2.26) is such a morphism.

ExAmple $(2.27)$. (a) Let $(\mathscr{X}, \mathscr{A})$ and $(\mathscr{Y}, \mathscr{A})$ be strict left (right) $\mathscr{R}$-pairs of spectra. Then the diagram

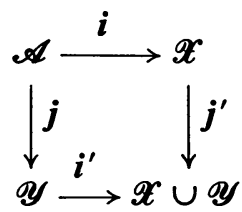

is a pushout for morphisms of strict left (right) $\mathscr{R}$-spectra. 
For each $n$, the diagram

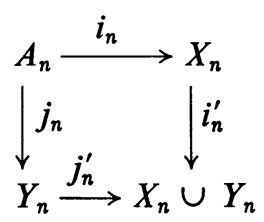

is a pushout in $Q^{\circ}$ since both $i_{n}$ and $j_{n}$ are cofibrations. As the pushout is unique, Proposition (2.23) then implies the conclusion.

(b) Let $\mathscr{R}$ be a strict ring spectrum, each of the maps

$$
* \rightarrow A \rightarrow X
$$

be a cofibration, and $\mathscr{M}$ be a strict right $\mathscr{R}$-spectrum. Then $(X \wedge \mathscr{M}, A \wedge \mathscr{M})$ is a strict right $\mathscr{R}$-pair. In particular, let $(T,\{0,1\},\{0\})$ be the triple consisting of the unit interval and the two indicated subsets. Then

$$
(T \wedge \mathscr{M},\{0,1\} \wedge \mathscr{M})=(T \mathscr{M}, \mathscr{M})
$$

is the pair consisting of the cone on $\mathscr{M}$ and $\mathscr{M}$; it is a strict right $\mathscr{R}$-pair.

Let $f: \mathscr{M} \rightarrow \mathscr{N}$ be a morphism of strict right $\mathscr{R}$-spectra. By Proposition (2.23) we have the commutative diagram

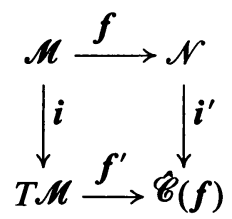

of strict right $\mathscr{R}$-morphisms which is a pushout in that category. Thus,

PROPOSITION (2.29). If $\mathscr{R}$ is a strict ring spectrum and $f: \mathscr{M} \rightarrow \mathscr{N}$ is a morphism of strict right $\mathscr{R}$-spectra, then $(\hat{\mathscr{C}}(\boldsymbol{f}), \mathscr{N})$ is a strict right $\mathscr{R}$-pair.

Returning to pairings, we have

Proposition (2.30). (1) If $t:((\mathscr{X}, \mathscr{A}),(\mathscr{Y}, *)) \rightarrow(\mathscr{Z}, \mathscr{C}, *)$ is a relative pairing then the diagrams

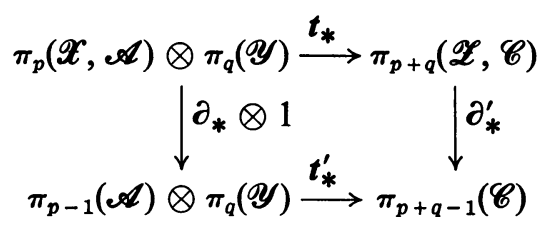

commute. 
(2) If $t:((\mathscr{X}, *),(\mathscr{Y}, \mathscr{B})) \rightarrow(\mathscr{Z}, \mathscr{C}, *)$ is a relative pairing, then the diagrams

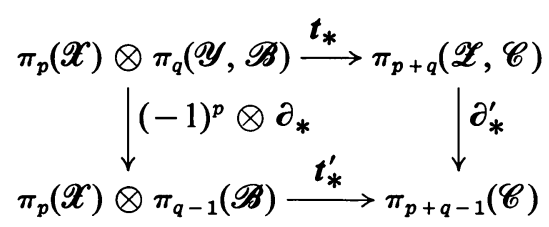

commute.

Proof. We give here only the proof of (2). For each pair of integers $(m, n)$, the diagrams

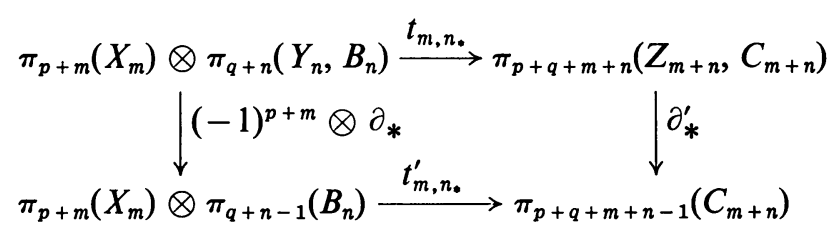

commute. The homomorphism $t_{*}$ is determined by $(-1)^{p n} t_{m, n}, \partial_{*}$ is determined by $(-1)^{n} \partial_{*}, \partial_{*}^{\prime}$ is determined by $(-1)^{m+n} \partial_{*}^{\prime}$, and $t_{*}^{\prime}$ is determined by $(-1)^{p n} t_{m, n_{*}}^{\prime}$ It follows that the diagrams of (2) commute.

An easy argument based on the previous proposition proves

COROllary (2.31). If

$$
\boldsymbol{t}:((\mathscr{X}, \mathscr{A}),(\mathscr{Y}, \mathscr{B})) \rightarrow(\mathscr{Z}, \mathscr{C}, \mathscr{D})
$$

is a relative pairing, the diagrams

$$
\begin{aligned}
& \pi_{p}(\mathscr{X}, \mathscr{A}) \otimes \pi_{p}(\mathscr{Y}, \mathscr{B}) \stackrel{\boldsymbol{t}_{*}}{\longrightarrow} \pi_{p+q}(\mathscr{Z}, \mathscr{C}) \\
& \downarrow\left(\partial_{*} \otimes 1\right) \oplus\left((-1)^{p} \otimes \partial_{*}\right) \\
&\left(\pi_{p-1}(\mathscr{A}) \otimes \pi_{q}(\mathscr{Y}, \mathscr{B})\right)\left.\right|_{*} \\
& \oplus\left(\pi_{p}(\mathscr{X}, \mathscr{A}) \otimes \pi_{q-1}(\mathscr{B})\right) \longrightarrow \pi_{p+q-1}(\mathscr{C}, \mathscr{D})
\end{aligned}
$$

commute.

ExAmple $(2.32)$. Let $(\mathscr{X}, \mathscr{A})$ be a right $\mathscr{R}$-pair of spectra; then $\pi_{*}(\mathscr{X}, \mathscr{A})$ $\cong \pi_{*}(\mathscr{X} / \mathscr{A})$ as right $\pi_{*}(\mathscr{R})$-modules. By $(2.30), \partial_{*}: \pi_{*}(\mathscr{X}, \mathscr{A}) \rightarrow \pi_{*}(\mathscr{A})$ is a homomorphism of degree $(-1)$ of right $\pi_{*}(\mathscr{R})$-modules. Thus, the exact sequence (2.15) is an exact sequence of right $\pi_{*}(\mathscr{R})$-modules.

Definition (2.33). Let $\mathscr{R}$ be a ring spectrum, $\mathscr{M}$ and $\mathscr{N}$ right and left $\mathscr{R}$ spectra, respectively, and

$$
\gamma:(\mathscr{M}, \mathscr{N}) \rightarrow \mathscr{P}
$$


a pairing. The pairing $\gamma$ is balanced over $\mathscr{R}$ provided the diagrams

$$
\begin{aligned}
M_{p} \wedge & R_{q} \wedge N_{r} \stackrel{\rho_{p, q} \wedge N_{r}}{\longrightarrow} M_{p+q} \wedge N_{r} \\
& \downarrow M_{p} \wedge \lambda_{q, r} \quad \downarrow \gamma_{p+q, r} \\
M_{p} & \wedge N_{q+r} \underset{\gamma_{p, q+r}}{\longrightarrow} P_{p+q+r}
\end{aligned}
$$

are homotopy commutative for each $p, q$ and $r$.

EXAMPLE (2.35). Any pairing is balanced over $\mathscr{S}$. If $\mathscr{M}$ is a right $\mathscr{R}$-spectrum, then $\gamma:(\mathscr{K}, \mathscr{R}) \rightarrow \mathscr{M}$ is balanced over $\mathscr{R}$.

Proposition (2.36). If the pairing $\gamma:(\mathscr{M}, \mathscr{N}) \rightarrow \mathscr{P}$ is balanced over the ring spectrum $\mathscr{R}$, then the induced homomorphism

$$
\gamma_{*}: \pi_{*}(\mathscr{M}) \otimes \pi_{*}(\mathscr{N}) \rightarrow \pi_{*}(\mathscr{P})
$$

is balanced over $\pi_{*}(\mathscr{R})$ and defines

$$
\gamma_{*}: \pi_{*}(\mathscr{M}) \underset{\pi_{*}(\mathscr{R})}{\bigotimes} \pi_{*}(\mathscr{N}) \rightarrow \pi_{*}(\mathscr{P})
$$

The proof is routine.

Definition (2.37). Let $\mathscr{R}$ be a ring spectrum and $(\mathscr{X}, \mathscr{A})$ and $(\mathscr{Y}, \mathscr{B})$ be right and left $\mathscr{R}$-pairs, respectively. A relative pairing

$$
\boldsymbol{t}:((\mathscr{X}, \mathscr{A}),(\mathscr{Y}, \mathscr{B})) \rightarrow(\mathscr{Z}, \mathscr{C}, \mathscr{D})
$$

is a balanced pairing of pairs of spectra over $\mathscr{R}$ provided $t:(\mathscr{X}, \mathscr{Y}) \rightarrow \mathscr{Z}$ is a balanced pairing over $\mathscr{R}$ such that the restrictions of the maps and homotopies making $t$ balanced over $\mathscr{R}$ also define balanced pairings over $\mathscr{R}:(\mathscr{X}, \mathscr{B}) \rightarrow \mathscr{C}$, $(\mathscr{A}, \mathscr{Y}) \rightarrow \mathscr{C}$ and $(\mathscr{A}, \mathscr{B}) \rightarrow \mathscr{D}$.

REMARK (2.38). A balanced pairing of pairs over $\mathscr{R} t:((\mathscr{X}, \mathscr{A}),(\mathscr{Y}, \mathscr{B})) \rightarrow$ $(\mathscr{Z}, \mathscr{C}, \mathscr{D})$ induces a balanced pairing over $\mathscr{R}$

$$
\boldsymbol{t}^{\prime}:(\mathscr{X} / \mathscr{A}, \mathscr{Y} / \mathscr{B}) \rightarrow \mathscr{Z} / \mathscr{C} \text {. }
$$

Proposition (2.39). Let $t:((\mathscr{X}, \mathscr{A}),(\mathscr{Y}, \mathscr{B})) \rightarrow(\mathscr{Z}, \mathscr{C}, \mathscr{D})$ be a balanced pairing of pairs of spectra over $\mathscr{R}$. Then

(1) $t$ induces

and

$$
t_{*}: \pi_{*}(\mathscr{X}, \mathscr{A}) \underset{\pi_{*}(\mathscr{R})}{\otimes} \pi_{*}(\mathscr{Y}, \mathscr{B}) \rightarrow \pi_{*}(\mathscr{Z}, \mathscr{C})
$$

(2) the diagrams

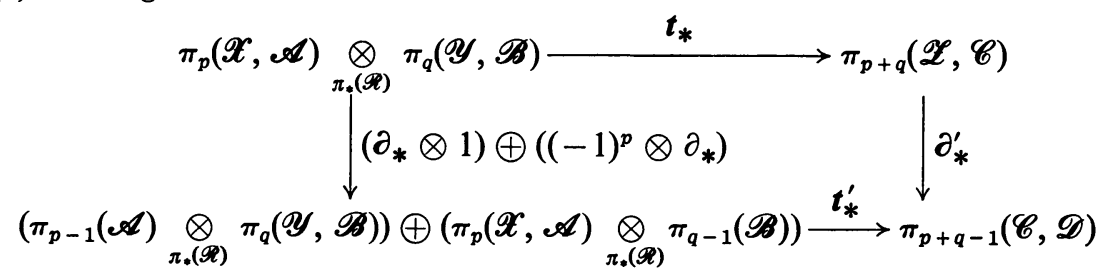

commute. 
Proof. (1) is an easy consequence of (2.31), (2.36) and (2.38). (2) follows from (2.30) and (2.36).

Let $H$ be a monoid and $\mathscr{A}$ (strict) ring spectrum. As was seen in (2.9c), $\mathscr{A} \wedge H^{+}=\mathscr{R}$ is a (strict) ring spectrum. If $X$ is a left $H$-space, then $\mathscr{A} \wedge X^{+}$is a (strict) left $\mathscr{R}$-spectrum.

For $X$ and $Y$ in $(Q H)^{\circ}$ and $(H Q)^{\circ}$ (Definition (1.7)), respectively, $X \wedge_{H} Y$ is the object of $Q^{\circ}$ obtained from $X \times{ }_{H} Y$ (Definition (1.2)) by identifying $X \times_{H} y_{0}$ $\cup x_{0} \times_{H} Y$ to a point and giving the decomposition set the quotient topology.

Proposition (2.40). Let $\mathscr{A}$ be a strict ring spectrum and $H$ a monoid. Let $\mathscr{R}=\mathscr{A} \wedge H^{+}, X$ be an object of $H Q$ and $\mathscr{M}$ be a strict right $\mathscr{R}$-spectrum. The maps $\gamma_{p, q}$ given by

$$
M_{p} \wedge\left(A_{q} \wedge X^{+}\right) \rightarrow M_{p+q} \wedge X^{+} \rightarrow M_{p+q} \wedge_{H} X^{+}
$$

define a pairing

$$
\gamma:\left(\mathscr{M}, \mathscr{A} \wedge X^{+}\right) \rightarrow \mathscr{M} \wedge_{H} X^{+}
$$

which is balanced over $\mathscr{R}$.

Proof. In order to prove that $\left\{\gamma_{p, q}\right\}$ is $\mathscr{R}$-balanced, we note that the commutativity of the diagrams (2.34) follows from that of the diagrams

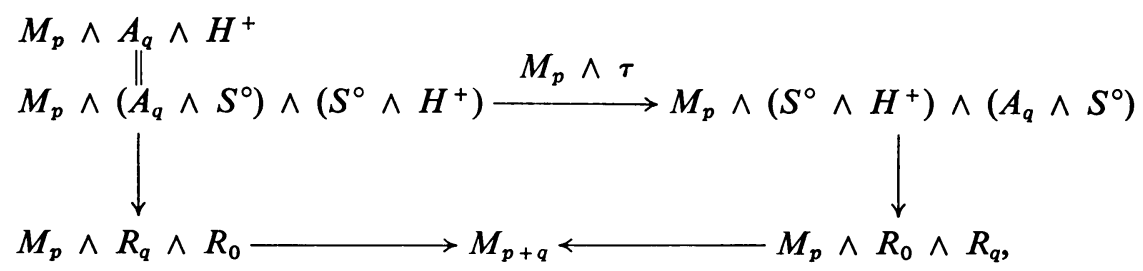

where $A_{q} \wedge S^{\circ} \rightarrow R_{q}$ is the composition

$$
A_{q} \wedge S^{\circ} \rightarrow A_{q} \wedge\{e,+\} \rightarrow A_{q} \wedge H^{+}=R_{q}
$$

and $S^{\circ} \wedge H^{+} \rightarrow R_{0}$ is the composition

$$
S^{\circ} \wedge H^{+} \stackrel{\mu_{0} \wedge H^{+}}{\longrightarrow} A_{0} \wedge H^{+}=R_{0} .
$$

That the diagrams (2.41) commute follows from condition (1) of Definition (2.10) and conditions (2) (for $i=0$ ) and (3) (for $j=0$ ) of Definition (2.7).

The fact that the maps $\left\{\gamma_{p, q}\right\}$ define a pairing follows immediately from the fact that $\mathscr{M}$ is a right $\mathscr{A}$-spectrum.

The diagrams (2.41) show that $\mathscr{M}$ is a strict right $\mathscr{A}$-spectrum, and rignt $H$ spectrum and that these two right actions commute. There are then unique functions

$$
\omega_{p, s}: M_{p} \wedge_{H} X^{+} \wedge A_{s} \rightarrow M_{p+s} \wedge_{H} X^{+}
$$


such that the diagrams

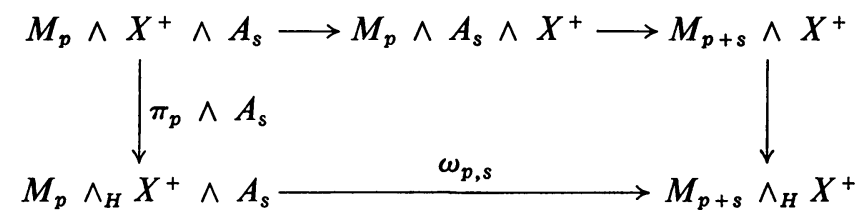

commute. Since $\pi_{p} \wedge A_{s}$ is a projection and $\omega_{p, s}\left(\pi_{p} \wedge A_{s}\right)$ is continuous, $\omega_{p, s}$ is continuous. Since $\mathscr{M}$ is a right $\mathscr{A}$-spectrum, these diagrams imply the family $\left\{\omega_{p, s}\right\}$ give $\mathscr{M} \wedge_{H} X^{+}$the structure of a right $\mathscr{A}$-spectrum. From the commutativity of the diagrams

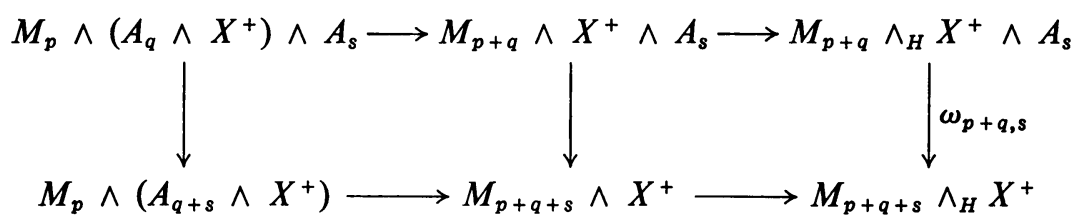

we can conclude the

Corollary (2.42). Under the hypotheses of Proposition (2.40), $\mathscr{M} \wedge_{H} X^{+}$is a right $\mathscr{A}$-spectrum and $\gamma_{*}: \pi_{*}(\mathscr{M}) \bigotimes_{\pi_{*}(\mathscr{R})} \pi_{*}\left(\mathscr{A} \wedge X^{+}\right) \rightarrow \pi_{*}\left(\mathscr{M} \wedge_{H} X^{+}\right)$is a homomorphism of right $\pi_{*}(\mathscr{A})$-modules.

Proposition (2.43). If, in addition to the hypotheses of Proposition (2.40), $\mathscr{M}=\bigvee_{\sigma \in \Sigma}\left(S^{n_{\sigma}} \wedge \mathscr{A} \wedge H^{+}\right)$, then $\pi_{*}(\mathscr{M})$ is a free right $\pi_{*}(\mathscr{R})$-module and

$$
\gamma_{*}: \pi_{*}(\mathscr{M}) \underset{\pi^{*}(\mathscr{R})}{\bigotimes} \pi_{*}\left(\mathscr{A} \wedge X^{+}\right) \rightarrow \pi_{*}\left(\mathscr{M} \wedge_{H} X^{+}\right)
$$

is an isomorphism of right $\pi_{*}(\mathscr{A})$-modules.

Proof. Since $\pi_{*}$ is stably additive on wedges, it suffices to prove the assertion for $\mathscr{M}=S^{n} \wedge \mathscr{A} \wedge H^{+}$.

The composition

$$
\begin{aligned}
S^{m} \wedge S^{p} \wedge(e,+) \wedge A_{q} \wedge X^{+} & \longrightarrow S^{m} \wedge S^{p} \wedge H^{+} \wedge A_{q} \wedge X^{+} \\
& \longrightarrow S^{m} \wedge A_{p+q} \wedge H^{+} \wedge X^{+} \\
& \longrightarrow S^{m} \wedge A_{p+q} \wedge H^{+} \wedge_{H} X^{+} \\
& \cong S^{m} \wedge A_{p+q} \wedge X^{+}
\end{aligned}
$$

shows $\gamma_{*}$ is onto.

$\pi_{*}(\mathscr{M})$ is the free $\pi_{*}(\mathscr{R})$-module generated by $g_{n}$, the class of the composition

$$
S^{n} \longrightarrow S^{n} \wedge S^{\circ} \wedge(e,+) \stackrel{S^{n} \wedge u_{0} \wedge i}{\longrightarrow} S^{n} \wedge A_{0} \wedge H^{+},
$$


where $i$ is the inclusion $(e,+) \subset H^{+}$. Thus, $\pi_{*}(\mathscr{M}) \otimes_{\pi_{*}(\mathscr{R})} \pi_{*}\left(\mathscr{A} \wedge X^{+}\right)$is isomorphic to $\pi_{*}\left(S^{n} \wedge \mathscr{A} \wedge X^{+}\right)$by the homomorphism

$$
\left(g_{n}, \boldsymbol{f}\right) \rightarrow i_{n} \wedge \boldsymbol{f}
$$

for $f \in \pi_{*}\left(\mathscr{A} \wedge X^{+}\right)$and $i_{n}=1_{S^{n}}: S^{n} \rightarrow S^{n}$.

But $\mathscr{M} \wedge_{H} X^{+} \cong S^{n} \wedge \mathscr{A} \wedge H^{+} \wedge_{H} X^{+} \cong S^{n} \wedge \mathscr{A} \wedge X^{+}$and for $f_{j}: S^{j} \rightarrow A_{p} \wedge X^{+}$, the class of $\gamma_{*}\left(g_{n},\left(f_{j}\right)\right)$ is the class of the composition

which is $i_{n} \wedge\left(f_{j}\right)$.

$$
\begin{aligned}
S^{n} \wedge S^{j} & \rightarrow S^{n} \wedge S^{\circ} \wedge(e,+) \wedge S^{j} \\
& \rightarrow S^{n} \wedge A_{0} \wedge H^{+} \wedge A_{p} \wedge X^{+} \\
& \rightarrow S^{n} \wedge A_{p} \wedge H^{+} \wedge X^{+} \\
& \rightarrow S^{n} \wedge A_{p} \wedge H^{+} \wedge X^{+} \\
& \rightarrow S^{n} \wedge A_{p} \wedge X^{+}
\end{aligned}
$$

III. Resolutions of spectra. In this section we construct resolutions of spectra which are analogous to and have the role of projective resolutions in homological algebra. As these lead directly to projective resolutions of homology, we have the conceptual simplification of avoiding the split projective resolutions used by Eilenberg and Moore, at the expense of somewhat involved spectra constructions.

Definition (3.1). For a strict ring spectrum $\mathscr{R}$, a strict left $\mathscr{R}$-spectrum $\mathscr{X}$, and a map $f: A \rightarrow X_{n}$ in $Q^{\circ}, \mathscr{T}(f)$ is the pushout defined by

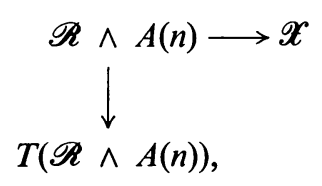

where, as before, $T$ is the cone functor and $\mathscr{R} \wedge A(n) \rightarrow \mathscr{X}$ is the morphism of strict left $\mathscr{R}$-spectra defined by the composition

$$
\mathscr{R} \wedge A(n) \rightarrow \mathscr{R} \wedge X_{n}(n) \rightarrow \mathscr{X} .
$$

We note that in Example (2.27) the left cone functor was discussed only for right $\mathscr{R}$-spectra. There is the homeomorphism

$$
T \wedge R_{i} \wedge A \stackrel{\tau \wedge A}{\longrightarrow} R_{i} \wedge T \wedge A,
$$

and the left $\mathscr{R}$-action on $T(\mathscr{R} \wedge A)$ is defined in the obvious way using this homeomorphism and Example (2.27).

As a consequence of Proposition (2.23), we have

LemMA (3.2). For $\mathscr{R}$ a strict ring spectrum, $\mathscr{X}$ a strict left $\mathscr{R}$-spectrum, and $f$ : $A \rightarrow X_{n}$ a $Q^{\circ}$-morphism, the pair $(\mathscr{T}(f), \mathscr{X})$ is a strict left $\mathscr{R}$-pair of spectra. 
Definition (3.3). Let $\mathscr{R}$ denote a strict ring spectrum, $\mathscr{X}$ a strict left $\mathscr{R}$-spectrum, and $(f, \Sigma)$ a family of maps

$$
f_{\sigma}: A_{\sigma} \rightarrow X_{n_{\sigma}}, \quad \sigma \in \Sigma,
$$

in $Q^{\circ}$. Then $\mathscr{T}(f, \Sigma)$ is the pushout of the diagram

$$
\mathscr{X} \stackrel{\boldsymbol{i}_{\sigma}}{\longrightarrow} \mathscr{T}\left(f_{\sigma}\right), \quad \sigma \in \Sigma .
$$

Again by Proposition (2.23), we have that $\mathscr{T}(f, \Sigma)$ is a strict left $\mathscr{R}$-spectrum and each $\mathscr{T}\left(f_{\sigma}\right) \rightarrow \mathscr{T}(f, \Sigma)$ is a strict left $\mathscr{R}$-pair. Since each $\boldsymbol{i}_{\sigma}$ is also a strict left $\mathscr{R}$-pair and a composition of cofibrations is a cofibration, we have

Proposition (3.4). For $\mathscr{R}$ a strict ring spectrum, $\mathscr{X}$ a strict left $\mathscr{R}$-spectrum, and $(f, \Sigma)$ a family of $Q^{\circ}$-morphisms

$$
f_{\sigma}: A_{\sigma} \rightarrow X_{n_{\sigma}}, \quad \sigma \in \Sigma,
$$

$(\mathscr{T}(f, \Sigma), \mathscr{X})$ is a strict left $\mathscr{R}$-pair of spectrum.

Definition (3.5). If, under the hypotheses of Proposition (3.4), each of the spaces $A_{\sigma}$ is a sphere, the pair $(\mathscr{T}(f, \Sigma), \mathscr{X})$ is said to be a relatively free left $\mathscr{R}$-pair.

The notion will have the role for spectra, when $\mathscr{R}=\mathscr{S}$, to adjoining stable cells.

Definition (3.6). Let $\mathscr{R}$ denote a strict ring spectrum.

(a) A left $\mathscr{R}$-complex is a sequence

$$
\mathscr{X}^{i} \rightarrow \mathscr{X}^{i+1} \rightarrow \mathscr{X}^{i+2} \rightarrow \cdots
$$

of strict left $\mathscr{R}$-spectra such that for each $j, i \leqq j,\left(\mathscr{X}^{j+1}, \mathscr{X}^{j}\right)$ is a relatively free left $\mathscr{R}$-pair.

(b) Let $\mathscr{R}$ denote a strict ring spectrum and $\mathscr{M}$ a strict left $\mathscr{R}$-spectrum. A resolution of $\mathscr{M}$ is a left $\mathscr{R}$-complex

$$
\mathscr{X}^{-1} \rightarrow \mathscr{X}^{0} \rightarrow \mathscr{X}^{1} \rightarrow \cdots
$$

with $\mathscr{M}=\mathscr{X}^{-1}$.

(c) The left $\mathscr{R}$-complex $\mathscr{X}^{i} \rightarrow \mathscr{X}^{i+1} \rightarrow \cdots$ is acyclic if for each $j, i \leqq j$, the homomorphism $\pi_{*}\left(\mathscr{X}^{j}\right) \rightarrow \pi_{*}\left(\mathscr{X}^{j+1}\right)$ is trivial.

(d) It is strongly acyclic if moreover for each $j$ and $n$, the homomorphism

is trivial.

$$
\pi_{*}\left(X_{n}^{j}\right) \rightarrow \pi_{*}\left(X_{n}^{j+1}\right)
$$

Lemma (3.7). For the strict ring spectrum $\mathscr{R}$, let $(\mathscr{X}, \mathscr{A})$ be a relatively free left $\mathscr{R}$-pair and $(\mathscr{Y}, \mathscr{B})$ be a strict left $\mathscr{R}$-pair such that each of the homomorphisms

$$
\pi_{*}\left(B_{n}\right) \rightarrow \pi_{*}\left(Y_{n}\right)
$$

is zero. Then each morphism $\mathscr{G}: \mathscr{A} \rightarrow \mathscr{B}$ of strict left $\mathscr{R}$-spectra has an extension to a morphism $\mathscr{G}^{\prime}: \mathscr{X} \rightarrow \mathscr{Y}$ of strict left $\mathscr{R}$-spectra. 
Proof. In the notation of Definition (3.3), for some $(f, \Sigma), f_{\sigma}: S^{m_{\sigma}} \rightarrow A_{n_{\sigma}}$, $\sigma \in \Sigma, \mathscr{X}=\mathscr{T}(f, \Sigma)$.

Each of the compositions

$$
S^{m_{\sigma}} \rightarrow A_{n_{\sigma}} \rightarrow B_{n_{\sigma}} \rightarrow Y_{n_{\sigma}}
$$

is null-homotopic and thus extends to a map

and a map

$$
\tilde{f}_{\sigma}: T S^{m_{\sigma}} \rightarrow Y_{n_{\sigma}}
$$

$$
\tilde{f}_{\sigma}: T\left(\mathscr{R} \wedge S^{m_{\sigma}}\right)\left(n_{\sigma}\right) \rightarrow \mathscr{Y}
$$

factoring through $\mathscr{R} \wedge Y_{n_{\sigma}}\left(n_{\sigma}\right)$. This yields the commutative diagram

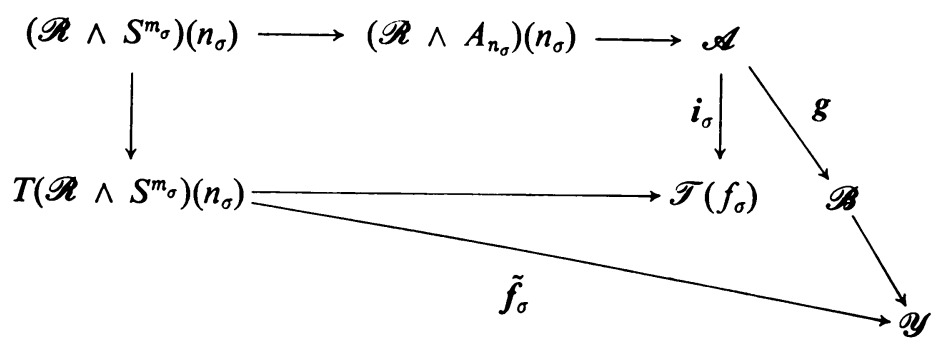

of strict left $\mathscr{R}$-morphisms, in which the square is a pushout. By Proposition (2.23), there exist a map $g_{\sigma}: \mathscr{T}\left(f_{\sigma}\right) \rightarrow \mathscr{Y}$ making the diagram commute. The compositions $\boldsymbol{g}_{\sigma} \boldsymbol{i}_{\sigma}$ are independent of $\sigma$ and thus define a morphism

extending $g$.

$$
\boldsymbol{g}^{\prime}: \mathscr{X} \rightarrow \mathscr{Y}
$$

LEMMA (3.8). If

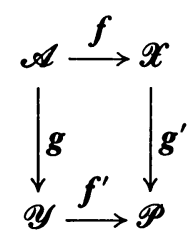

is a pushout of morphisms of strict left $\mathscr{R}$-spectra, $\mathrm{g}$ is a cofibration, and $h: C \rightarrow Z$ is a cofibration in $Q^{\circ}$, then

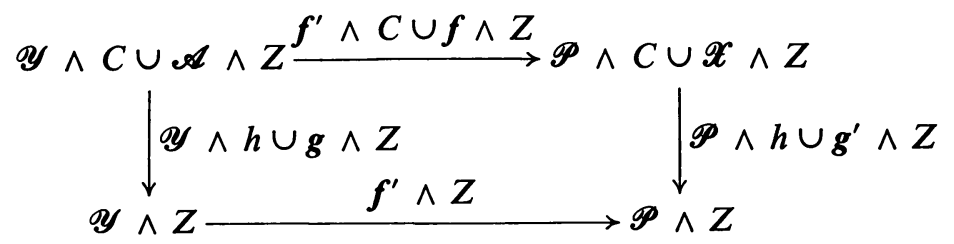

is a pushout of morphisms of strict left $\mathscr{R}$-spectra. 
Proof. The diagram

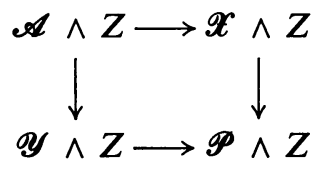

is such a pushout. It can be factored as follows:

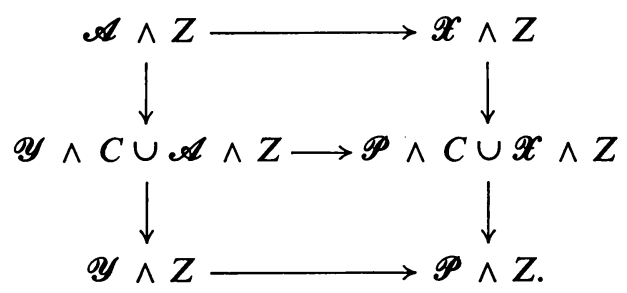

The conclusion of the lemma then is implied by the fact that the upper square is a pushout. This implication is a property of pushouts in any category.

Suppose $p: \mathscr{Y} \wedge C \cup \mathscr{A} \wedge Z \rightarrow \mathscr{W}$ and $q: \mathscr{X} \wedge Z \rightarrow \mathscr{W}$ are morphisms of strict left $\mathscr{R}$-spectra such that $p i=q f \wedge Z$. Then

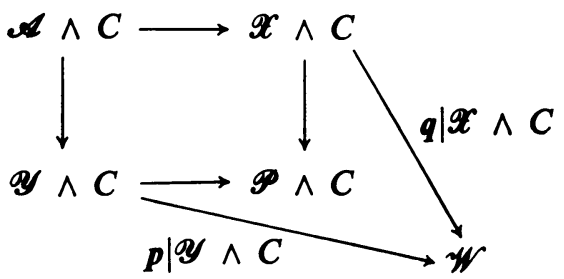

commutes and defines a unique morphism $r: \mathscr{P} \wedge C \rightarrow \mathscr{W}$ of strict left $\mathscr{R}$-spectra such that $r f^{\prime} \wedge C=p \mid y \wedge C$ and $r g^{\prime} \wedge C=q \mid \mathscr{X} \wedge C$. The diagram

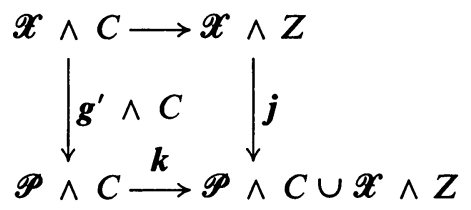

is a pushout. Also, $\boldsymbol{r} g^{\prime} \wedge C=q \mid \mathscr{X} \wedge C=q \mathscr{X} \wedge h$. Thus there is a unique morphism $s: \mathscr{P} \wedge C \cup \mathscr{X} \wedge Z \rightarrow \mathscr{W}$ of strict left $\mathscr{R}$-spectra such that

Thus,

$$
s j=q \text { and } s k=r .
$$

$$
\begin{aligned}
s\left(\boldsymbol{f}^{\prime} \wedge C \cup \boldsymbol{f} \wedge Z\right) \mid \boldsymbol{y} \wedge C & =\boldsymbol{s} \boldsymbol{k} \boldsymbol{f}^{\prime} \wedge C \\
& =\boldsymbol{r} \boldsymbol{f}^{\prime} \wedge C \\
& =\boldsymbol{p} \mid \boldsymbol{y} \wedge C,
\end{aligned}
$$


and

$$
\begin{aligned}
s\left(f^{\prime} \wedge C \cup f \wedge Z\right) \mid \mathscr{A} \wedge Z & =s j f \wedge Z \\
& =q f \wedge Z \\
& =p i .
\end{aligned}
$$

Consequently, $s\left(f^{\prime} \wedge C \cup f \wedge Z\right)=p$.

Any $\tilde{\boldsymbol{s}}: \mathscr{P} \wedge C \cup \mathscr{X} \wedge Z \rightarrow \mathscr{W}$ such that $\tilde{\boldsymbol{s}}\left(\boldsymbol{f}^{\prime} \wedge C \cup \boldsymbol{f} \wedge Z\right)=\boldsymbol{p}$ and $\tilde{\boldsymbol{s}} \boldsymbol{j}=\boldsymbol{q}$ has restriction $r$ on $p \wedge C$ and so equals $s$.

Lemma (3.9). If $(\mathscr{X}, \mathscr{A})$ is a relatively free left $\mathscr{R}$-pair, then so is

$$
\left(\mathscr{X} \wedge I^{+}, \mathscr{X} \wedge S^{0^{+}} \cup \mathscr{A} \wedge I^{+}\right) .
$$

Proof. Let $\left(f_{\sigma}, \sigma \in \Sigma\right)$ be the defining family for $\mathscr{X}$. Let $Y_{n_{\sigma}}$ be the pushout of

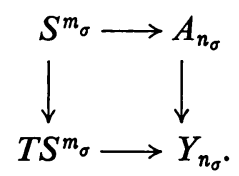

By definition $\mathscr{T}\left(f_{\sigma}\right)$ is the pushout

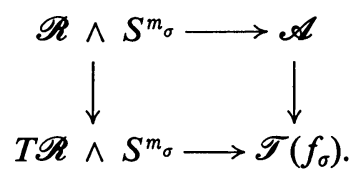

But this diagram factors:

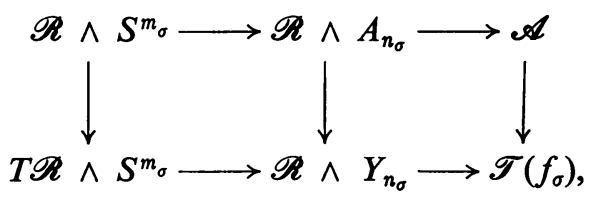

and the left square is also a pushout. Thus, the right square is a pushout. Also the square

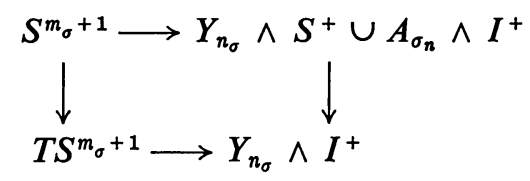

is a pushout, where $S=S^{0}=\partial I$.

Define $\mathscr{Z}_{\sigma}$ to be the pushout of

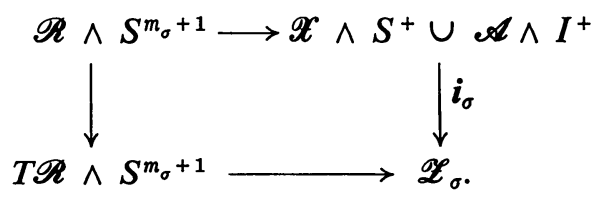

We wish to show that $\mathscr{X} \wedge I^{+}$is the pushout of $\mathscr{X} \wedge S^{+} \cup \mathscr{A} \wedge I^{+} \stackrel{i_{\sigma}}{\rightarrow} \mathscr{Z}_{\sigma}, \sigma \in \Sigma$. 
The square above factors

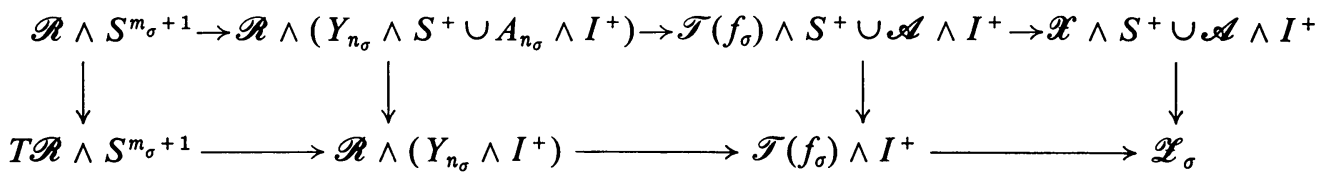

in which the first two squares are pushouts. Thus,

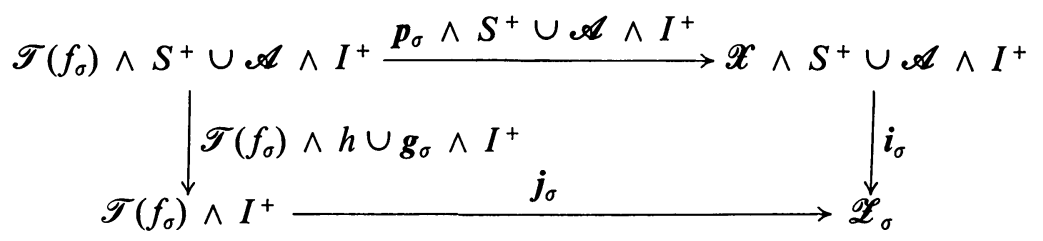

is a pushout. This defines morphisms

$$
\boldsymbol{q}_{\sigma}: \mathscr{Z}_{\sigma} \rightarrow \mathscr{X} \wedge I^{+}
$$

is strict left $\mathscr{R}$-spectra such that $q_{\sigma} i_{\sigma}$ is the inclusion $\mathscr{X} \wedge S^{+} \cup \mathscr{A} \wedge I^{+} \stackrel{i}{\rightarrow} \mathscr{X} \wedge I^{+}$ and $\boldsymbol{q}_{\sigma} \boldsymbol{j}_{\sigma}$ is the inclusion $\boldsymbol{p}_{\sigma} \wedge I^{+}$.

Suppose $f_{\sigma}: \mathscr{Z}_{\sigma} \rightarrow \mathscr{W}, \sigma \in \Sigma$, is a family of morphisms of strict left $\mathscr{R}$-spectra such that $\boldsymbol{f}_{\sigma} \boldsymbol{i}_{\sigma}$ is independent of $\sigma$. The compositions $\left(\boldsymbol{f}_{\sigma} \boldsymbol{j}_{\sigma}\right)\left(\boldsymbol{g}_{\sigma} \wedge I^{+}\right)$are then independent; and so, there is a unique morphism

$$
f: \mathscr{X} \wedge I^{+} \rightarrow \mathscr{W}
$$

such that $f p_{\sigma} \wedge I^{+}=f_{\sigma} j_{\sigma}$ for all $\sigma \in \Sigma$. To see that $f \boldsymbol{q}_{\sigma}=\boldsymbol{f}_{\sigma}$ for all $\sigma \in \Sigma$, we observe that

and

$$
\boldsymbol{f} \boldsymbol{q}_{\sigma} \boldsymbol{j}_{\sigma}=\boldsymbol{f} \boldsymbol{p}_{\sigma} \wedge I^{+}=\boldsymbol{f}_{\sigma} \boldsymbol{j}_{\sigma}
$$

$$
f \boldsymbol{q}_{\sigma} \boldsymbol{i}_{\sigma}=\boldsymbol{f} \mid \mathscr{X} \wedge S^{+} \cup \mathscr{A} \wedge I^{+} .
$$

However, $\boldsymbol{f} \mid \mathscr{X} \wedge S^{+} \wedge \mathscr{A} \wedge I^{+}$is the unique morphism $\boldsymbol{h}$ such that

$$
\boldsymbol{h} \boldsymbol{p}_{\sigma} \wedge S^{+} \cup \boldsymbol{j} \wedge I^{+}=\boldsymbol{f}_{\sigma} \boldsymbol{j}_{\sigma}\left(\mathscr{T}\left(f_{\sigma}\right) \wedge h \cup \boldsymbol{g}_{\sigma} \wedge I^{+}\right) .
$$

Since $\boldsymbol{f}_{\sigma} \boldsymbol{i}_{\sigma}$ is such a morphism,

Thus, $f \boldsymbol{q}_{\sigma}=\boldsymbol{f}_{\sigma}$.

$$
\boldsymbol{f q}_{\sigma} \boldsymbol{i}_{\sigma}=\boldsymbol{f}_{\sigma} \boldsymbol{i}_{\sigma}
$$

If $\tilde{f}: \mathscr{X} \wedge I^{+} \rightarrow \mathscr{W}$ is any morphism of strict left $\mathscr{R}$-spectra such that

$$
\tilde{f} \boldsymbol{q}_{\sigma}=\boldsymbol{f}_{\sigma} \text { for all } \boldsymbol{\sigma} \in \Sigma,
$$

then

and so $\tilde{\boldsymbol{f}}=\boldsymbol{f}$.

$$
\tilde{f} \boldsymbol{p}_{\sigma} \wedge I^{+}=\tilde{f} \boldsymbol{q}_{\sigma} \boldsymbol{j}_{\sigma}=\boldsymbol{f}_{\sigma} \boldsymbol{j}_{\sigma}
$$


THEOREM (3.10). Let $\mathscr{R}$ be a strict ring spectrum, $\varphi: \mathscr{M} \rightarrow \mathscr{N}$ be a morphism of strict left $\mathscr{R}$-spectra, $\left(\mathscr{X}^{i}\right)$ be a resolution of $\mathscr{M}$, and $\left(\mathscr{Y}^{i}\right)$ be a strongly acyclic resolution of $\mathscr{N}$. Then there exists a family of morphisms

$$
\boldsymbol{\Phi}^{i}: \mathscr{X}^{i} \rightarrow \mathscr{Y}^{i}, \quad-1 \leqq i,
$$

of strict left $\mathscr{R}$-spectra such that $\Phi^{-1}=\varphi$ and for each $j,-1 \leqq j$, the diagram

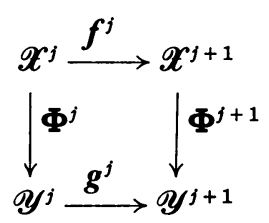

commutes.

If $\rho: \mathscr{M} \rightarrow \mathscr{N}$ and $\mathscr{H}^{-1}: \mathscr{X}^{-1} \wedge I^{+} \rightarrow \mathscr{Y}^{0}$ are morphisms of strict left $\mathscr{R}$-spectra such that

$$
\begin{aligned}
& \mathscr{H}^{-1} \mid \mathscr{X}^{-1} \wedge 0=\mathscr{G}^{-1} \varphi \\
& \mathscr{H}^{-1} \mid \mathscr{X}^{-1} \wedge 1=\mathscr{G}^{-1} \rho,
\end{aligned}
$$

and $\rho^{i}: \mathscr{X}^{i} \rightarrow \mathscr{Y}^{i},-1 \leqq i$, is a family of morphisms extending $\rho$, then there exist a family of morphisms

$$
\mathscr{H}^{i}: \mathscr{X}^{i} \wedge I^{+} \rightarrow \mathscr{Y}^{i+1}, \quad-1 \leqq i,
$$

such that

$$
\begin{aligned}
& \mathscr{H}^{i} \mid \mathscr{X}^{i} \wedge 0=\mathscr{E}^{i} \Phi^{i}, \\
& \mathscr{H}^{i} \mid \mathscr{X}^{i} \wedge 1=\mathscr{G}^{i} \rho^{i},
\end{aligned}
$$

and

$$
\mathscr{H}^{i} f^{i+1} \wedge I^{+}=\mathscr{G}^{i} \mathscr{H}^{i-1}
$$

Proof. The existence of a family $\left(\Phi^{i}\right)$ having the asserted properties is an immediate consequence of Lemma (3.7).

Suppose $\left(\rho^{i}\right)$ and $\mathscr{H}^{-1}$ are given as in the hypothesis. By Lemma (3.9),

$$
\left(\mathscr{X}^{0} \wedge I^{+}, \mathscr{X}^{0} \wedge S^{0+} \cup \mathscr{X}^{-1} \wedge I^{+}\right)
$$

is a relatively free left $\mathscr{R}$-pair. Then by Lemma (3.8), the morphism

has an extension

$$
\mathscr{G}^{0}\left(\boldsymbol{\Phi}^{0} \wedge 0 \cup \mathscr{H}^{-1} \cup \rho^{0} \wedge 1\right)
$$

$$
\mathscr{H}^{0}: \mathscr{X}^{0} \wedge I^{+} \rightarrow \mathscr{Y}^{1}
$$

This process clearly continues inductively.

THEOREM (3.11). Each strict left $\mathscr{R}$-spectrum has a strongly acyclic resolution.

Proof. For each $n$, let

$$
f_{\sigma}: S^{n_{\sigma}} \rightarrow M_{n}, \quad \sigma \in \Sigma_{n}
$$


be a family of maps generating $\pi_{*}\left(M_{n}\right)$. Let $\Sigma=\bigcup_{n} \Sigma_{n}$ and $\mathscr{X}^{0}=\mathscr{T}(f, \Sigma)$. By definition, $\left(\mathscr{X}^{0}, \mathscr{X}^{-1}\right)$ is a relatively free left $\mathscr{R}$-pair, where $\mathscr{M}=\mathscr{X}^{-1}$. Also, since each $X_{n}^{0}$ is the pushout of

$$
M_{n} \rightarrow \mathscr{T}_{n}\left(f_{\sigma}\right), \quad \sigma \in \Sigma_{n}
$$

and there are sufficiently many $f_{\sigma}$ to generate $\pi_{*}\left(M_{n}\right)$, the map $M_{n} \rightarrow \mathscr{T}(f, \Sigma)_{n}$ is trivial on homotopy groups.

Continue inductively forming $\mathscr{X}^{i+1}$ from $\mathscr{X}^{i}$ as was $\mathscr{X}^{0}$ formed from $\mathscr{X}^{-1}$. This yields a strongly acyclic resolution.

TheOREM (3.12). If $\mathscr{X}^{-1} \rightarrow \mathscr{X}^{0} \rightarrow \mathscr{X}^{1} \rightarrow \cdots$ is an acyclic resolution of the strict left $\mathscr{R}$-spectrum $\mathscr{M}$, then the sequence

$$
\pi_{*}(\mathscr{M}) \stackrel{\partial_{*}}{\longleftarrow} \pi_{*}\left(\mathscr{X}^{0}, \mathscr{M}\right) \stackrel{\partial_{*}}{\longleftarrow} \pi_{*}\left(\mathscr{X}^{1}, \mathscr{X}^{0}\right) \longleftarrow \cdots
$$

is a free left $\pi_{*}(\mathscr{R})$-resolution of $\pi_{*}(\mathscr{M})$.

Proof. In the notation of the previous proof we have the isomorphisms

$$
\pi_{*}\left(T\left(\mathscr{R} \wedge S^{n_{\sigma}}\right), \mathscr{R} \wedge S^{n_{\sigma}}\right) \stackrel{\cong}{\longrightarrow} \pi_{*}\left(\mathscr{T}\left(f_{\sigma}\right), \mathscr{M}\right)
$$

and

$$
\pi_{*}\left(T\left(\mathscr{R} \wedge S^{n_{\sigma}}\right), \mathscr{R} \wedge S^{n_{\sigma}}\right) \stackrel{\cong}{\longrightarrow} \pi_{*}\left(S^{1} \wedge \mathscr{R} \wedge S^{n_{\sigma}}\right)
$$

The latter is a free left $\pi_{*}(\mathscr{R})$-module on one generator. But

$$
\begin{aligned}
\pi_{*}\left(\mathscr{X}^{0}, \mathscr{M}\right) & =\pi_{*}(\mathscr{T}(f, \Sigma), \mathscr{M}) \\
& \cong \bigoplus_{\sigma \in \Sigma} \pi_{*}\left(\mathscr{T}\left(f_{\sigma}\right), \mathscr{M}\right)
\end{aligned}
$$

as left $\pi_{*}(\mathscr{R})$-modules. Thus, $\pi_{*}\left(\mathscr{X}^{0}, \mathscr{M}\right)$, and similarly $\pi_{*}\left(\mathscr{X}^{i+1}, \mathscr{X}^{i}\right)$, is a free left $\pi_{*}(\mathscr{R})$-module.

The remainder of the conclusion follows from the diagram

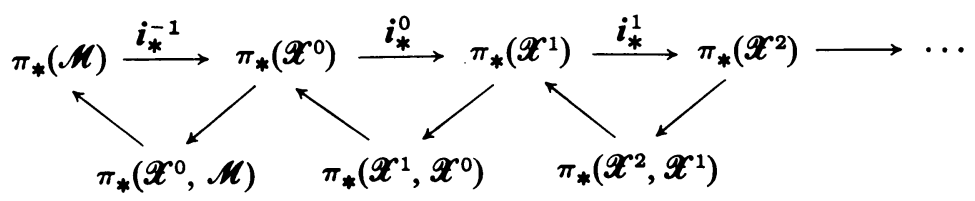

in which each triangle is an exact triangle of left $\pi_{*}(\mathscr{R})$-modules and each morphism $i_{*}^{k}$ is the zero homomorphism.

IV. The spectral sequence. Recall that in $\S I$ we defined the notions "right $H$-fibration" and "strong right $H$-fibration" (equally left) so that when the monoid $H$ is the trivial monoid $e$, these notions become the usual notions of Dold and Hurewicz fibration.

DEFINITION (4.1). A map $p: X \rightarrow B$ is a (strong) principal right $H$-bundle provided (a) $p$ is a (strong) right $H$-fibration and 
(b) for each path component $B_{0}$ of $B$, there exist a point $b \in B_{0}$ and a right $H$-equivalent homotopy equivalence of $p^{-1} b$ with $H$.

EXAMPLE (4.2). If for the map $p: X \rightarrow B$ with $X$ an object of $Q H$, the space $B$ has a numerable covering $\left\{U_{\sigma}\right\}_{\sigma \in \Sigma}$ such that for each $\sigma \in \Sigma, p^{-1} U_{\sigma}$ is homeomorphic to $U_{\sigma} \times H$ by a $Q H$-morphism over $U_{\sigma}$, then $p$ is a strong principal right $H$-bundle. If instead there is only a right $H$-equivariant fiber homotopy equivalence of $p^{-1} U_{\sigma}$ with $U_{\sigma} \times H$, then $p$ is a principal right $H$-bundle.

If $B$ is locally contractible in the large and $p: X \rightarrow B$ is a principal right $H$ bundle, then the converse holds. Locally contractible in the large is defined to mean that for some numerable covering $\left\{U_{\sigma}\right\}$ of $B$, (see Dold [6]) each injection $i_{\sigma}: U_{\sigma} \rightarrow B$ is null-homotopic.

Our principal result is

THEOREM (4.3). Let $\mathscr{A}$ be a strict ring spectrum, $H$ a monoid, F a $Q H^{\circ}$-space, and $p: X \rightarrow B$ a principal left $H$-bundle. Then there exists a right $h_{*}($ pt., $\mathscr{A})$-spectral sequence $E^{r}(p, H, F)$ and a filtration

$$
0=B_{-1, n+1} \subset B_{0, n} \subset \cdots
$$

of $\tilde{h}_{n}\left(F \wedge_{H} X^{+} ; \mathscr{A}\right)$ such that

(1) $E_{p, q}^{\prime}=0$ for $p<0$,

(2) $d_{p, q}^{r}: E_{p, q}^{r} \rightarrow E_{p-r, q+r-1}^{r}$,

(3) $\lim _{r} E_{p, q}^{r} \cong E_{p, q}^{\infty}$, where $E_{p, q}^{\infty}=B_{p, q} / B_{p-1, q+1}$,

(4) $\bigcup_{p} B_{p, n-p}=\tilde{h}_{n}\left(F \wedge_{H} X^{+} ; \mathscr{A}\right)$,

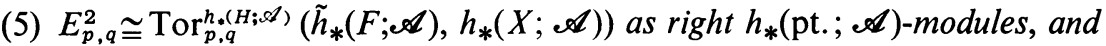

(6) the spectral sequence, filtration, and isomorphism (5) are natural with rexpect to

(a) morphisms $H \rightarrow H^{\prime}$ of monoids,

(b) morphisms $F \rightarrow F^{\prime}$ in $Q H^{\circ}$,

(c) morphisms of principal left $H$-bundles, and

(d) morphisms $\mathscr{A} \rightarrow \mathscr{B}$ of strict ring spectra.

We shall use

Lemma (4.4). Suppose $p: X \rightarrow B$ is a principal left $H$-bundle and $N$ is an object in $Q H^{\circ}$ such that $\pi_{*}(N)=0$. Then $\pi_{*}\left(N \wedge_{H} X^{+}\right)=0$.

Proof. $\left(N \times_{H} X, * \times_{H} X, \hat{p}, B\right)$ is a relative fibration with relative fiber $(N, *)$ and $* \times_{H} X \rightarrow N \times_{H} X$ is a cofibration (Proposition 1.6). The commutative diagram

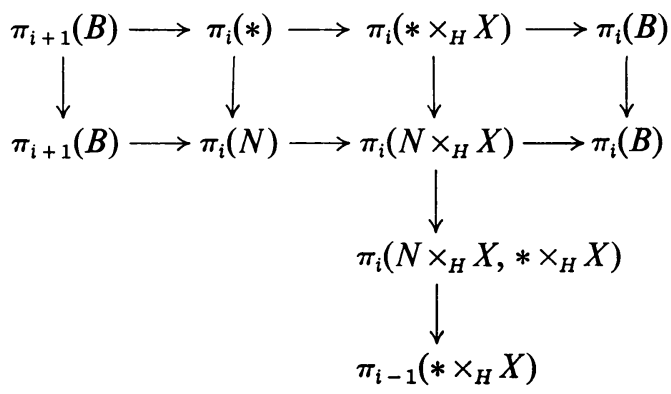


of exact homotopy sequences of the fibrations and of the pair $\left(N \times_{H} X, * \times_{H} X\right)$ implies

$$
\pi_{i}(N) \rightarrow \pi_{i}\left(N \times_{H} X, * \times_{H} X\right)
$$

is an isomorphism. Thus, the groups $\pi_{*}\left(N \times_{H} X, * \times_{H} X\right)$ are zero. The conclusion then follows from a Blakers-Massey theorem [4] and the fact that the map $* \times_{H} X \rightarrow N \times_{H} X$ is a cofibration.

Proof of Theorem (4.3). The spectrum $\mathscr{A} \wedge F$ is a strict right $\mathscr{A} \wedge H^{+}$spectrum. Let

$$
\mathscr{A} \wedge F=\mathscr{X}^{-1} \rightarrow \mathscr{X}^{0} \rightarrow \mathscr{X}^{1} \rightarrow \cdots
$$

be a strongly acyclic resolution over $\mathscr{A} \wedge H^{+}$. Let $\mathscr{X}=$ colim $\mathscr{X}^{i}$. Since for each $i$ and $k, \pi_{*}\left(X_{k}^{i}\right) \rightarrow \pi_{*}\left(X_{k}^{i+1}\right)$ is trivial, $\pi_{*}\left(X_{k}\right)=0$. Thus, $\pi_{*}\left(X_{k} \wedge_{H} X^{+}\right)=0$ by the previous lemma and $\pi_{*}\left(\mathscr{X} \wedge_{H} X^{+}\right)=0$. Let $\mathscr{Y}=\mathscr{X} / \mathscr{X}^{-1}$ and $\mathscr{Y}^{p}=\mathscr{X}^{p} / \mathscr{X}^{-1}$. Then

$$
\pi_{*}\left(\mathscr{Y} \wedge_{H} X^{+}\right) \cong \pi_{*}\left(\mathscr{X} \wedge_{H} X^{+}, \mathscr{X}^{-1} \wedge_{H} X^{+}\right) \underset{\cong}{\stackrel{\partial}{\longrightarrow}} \pi_{*}\left(\mathscr{X}^{-1} \wedge_{H} X^{+}\right)
$$

Define

$$
\begin{aligned}
& E_{p, q}^{r} \equiv \operatorname{Im}\left\{\pi_{p+q+1}\left(\mathscr{Y}^{p} \wedge_{H} X^{+}, \mathscr{Y}^{p-r} \wedge_{H} X^{+}\right)\right. \\
&\left.\rightarrow \pi_{p+q+1}\left(\mathscr{Y}^{p+r-1} \wedge_{H} X^{+}, \mathscr{Y}^{p-1} \wedge_{H} X^{+}\right)\right\} \\
& R_{p, q} \equiv \pi_{p+q+1}\left(\mathscr{Y}^{p} / \mathscr{Y}^{p-1}\right) \cong \pi_{p+q+1}\left(\mathscr{X}^{p} / \mathscr{X}^{p-1}\right), \quad p \geqq 0 \\
& \tilde{B}_{p, q} \equiv \operatorname{Im}\left\{\pi_{p+q+1}\left(\mathscr{Y}^{p} \wedge_{H} X^{+}\right) \rightarrow \pi_{p+q+1}\left(\mathscr{Y} \wedge_{H} X^{+}\right)\right\}
\end{aligned}
$$

and

$$
\begin{aligned}
B_{p, q} \equiv \operatorname{Im}\left\{\pi_{p+q+1}\left(\mathscr{Y}^{p} \wedge_{H} X^{+}\right) \longrightarrow \pi_{p+q+1}\left(\mathscr{Y} \wedge_{H} X^{+}\right)\right. & \stackrel{\partial}{\cong} \\
& \left.\cong \pi_{p+q}\left(X^{-1} \wedge_{H} X^{+}\right)\right\} .
\end{aligned}
$$

Theorem (3.10) implies the naturality asserted in conclusion (6) for the spectral sequence and filtration. Corollary (2.42) implies the spectral sequence is a right $h_{*}($ pt., $\mathscr{A})$-spectral sequence.

Since $\partial_{*}: \pi_{*}\left(\mathscr{Y} \wedge_{H} X^{+}\right) \rightarrow \pi_{*}\left(\mathscr{X}^{-1} \wedge_{H} X^{+}\right)$is an isomorphism, it induces an isomorphism of the filtered system $\left\{\widetilde{B}_{p, q}\right\}_{p+q=n}$ onto the filtered system $\left\{\boldsymbol{B}_{p, q}\right\}_{p+q}$. We have

$$
0=B_{-1, n+1} \subset B_{0, n} \subset \cdots \subset B_{p, q} \subset B_{p+1, q-1} \subset \ldots \subset \pi_{n}\left(\mathscr{A} \wedge F \wedge_{H} X^{+}\right),
$$

for $p+q=n$.

Any element of $\pi_{n+1}\left(\mathscr{Y} \wedge_{H} X^{+}\right)$can be represented by a map

$$
S^{n+1+i} \rightarrow Y_{i} \wedge_{H} X^{+}
$$


There is a commutative diagram

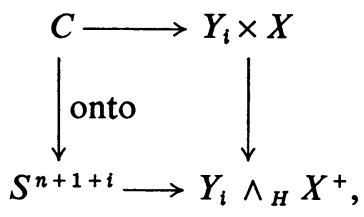

where $C$ is a compact, Hausdorff space. Since $Y_{i} \times X=\bigcup_{n} Y_{i}^{n} \times X, Y_{i}^{n} \subset Y_{i}^{n+1}$, and $C$ is compact, the diagram factors as follows

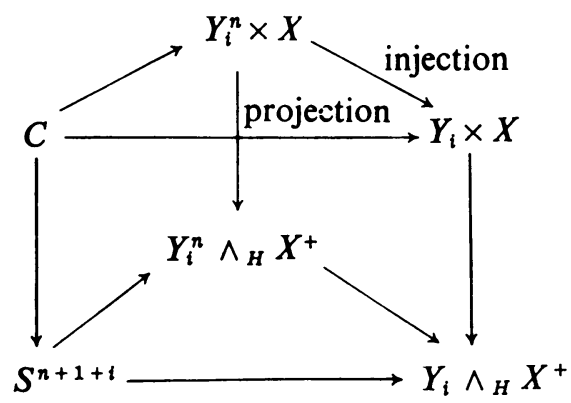

This shows that $\bigcup_{p} \widetilde{B}_{p, n-p}=\pi_{n+1}\left(\mathscr{Y} \wedge_{H} X^{+}\right)$, and consequently that

$$
\bigcup_{p} B_{p, n-p}=\pi_{n}\left(\mathscr{A} \wedge F \wedge_{H} X^{+}\right)
$$

which by definition is $\tilde{h}_{n}\left(F \wedge_{H} X^{+} ; \mathscr{A}\right)$.

$$
\begin{aligned}
& \text { For } r^{\prime} \geqq r \geqq p, \mathscr{Y}^{p-r} \wedge_{H} X^{+}=\mathscr{Y}^{p-r^{\prime}} \wedge_{H} X^{+}=* \text {, and } \\
& \pi_{p+q+1}\left(\mathscr{Y}^{p} \wedge_{H} X^{+}, *\right) \longrightarrow \pi_{p+q+1}\left(\mathscr{Y}^{p+r-1} \wedge_{H} X^{+}, \mathscr{Y}^{p-1} \wedge_{H} X^{+}\right) \\
& \pi_{p+q+1}\left(\mathscr{Y}^{p+r^{\prime}-1} \wedge_{H} X^{+}, \mathscr{Y}^{p-1} \wedge_{H} X^{+}\right) .
\end{aligned}
$$

Thus, $E_{p, q}^{r} \rightarrow E_{p, q}^{r^{\prime}}$ is onto for $r^{\prime} \geqq r \geqq p$.

Let $\bar{E}_{p, q}^{\infty}=\operatorname{Lim} E_{p, q}^{r}$. We compare this to $E_{p, q}^{\infty} \equiv B_{p, q} / B_{p-1, q+1} \cong \widetilde{B}_{p, q} / \widetilde{B}_{p-1, q+1}$. In the previous paragraph we showed

$$
\pi_{p+q+1}\left(\mathscr{Y} \wedge_{H} X^{+}\right)=\underset{\rightarrow}{\operatorname{Lim}} \pi_{p+q+1}\left(\mathscr{Y}^{p+r+1} \wedge_{H} X^{+}\right)
$$

Thus, we have

$$
\bar{E}_{p, q}^{\infty}=\operatorname{Im}\left\{\pi_{p+q+1}\left(\mathscr{Y}^{p} \wedge_{H} X^{+}\right) \rightarrow \pi_{p+q+1}\left(\mathscr{Y} \wedge_{H} X^{+}, \mathscr{Y}^{p-1} \wedge_{H} X^{+}\right)\right\} .
$$

This homomorphism factors by

$$
\pi_{p+q+1}\left(\mathscr{Y}^{p} \wedge_{H} X^{+}\right) \rightarrow \pi_{p+q+1}\left(\mathscr{Y} \wedge_{H} X^{+}\right) \rightarrow \pi_{p+q+1}\left(\mathscr{Y} \wedge_{H} X^{+}, \mathscr{Y}^{p-1} \wedge_{H} X^{+}\right),
$$

which defines an epimorphism

$$
\widetilde{B}_{p, q} \rightarrow \bar{E}_{p, q}^{\infty}
$$


Clearly, the kernel of this epimorphism is $\widetilde{B}_{p-1, q+1}$. Thus $E_{p, q}^{\infty} \stackrel{\cong}{\rightarrow} \bar{E}_{p, q}^{\infty}$. This completes the demonstration of all parts of the conclusion on Theorem (4.3) except (5).

To compute $E_{p, q}^{2}$ we observe that

$$
\begin{aligned}
E_{p, q}^{1}=\pi_{p+q+1}\left(\mathscr{Y}^{p} \wedge_{H} X^{+}, \mathscr{Y}^{p-1} \wedge_{H} X^{+}\right) & \cong \pi_{p+q+1}\left(\mathscr{Y}^{p} / \mathscr{Y}^{p-1} \wedge_{H} X^{+}\right) \\
& \cong \pi_{p+q+1}\left(\mathscr{X}^{p} / \mathscr{X}^{p-1} \wedge_{H} X^{+}\right)
\end{aligned}
$$

and that since $\mathscr{X}^{p} / \mathscr{X}^{p-1}=\bigvee_{\sigma}\left(S^{n_{\sigma}} \wedge \mathscr{A} \wedge H^{+}\right)$, by Proposition (2.43), there is an isomorphism

$$
\gamma_{*}^{p}: R_{p, *} \bigotimes_{\pi_{0}\left(\mathscr{A} \wedge H^{+}\right)} \pi_{*}\left(\mathscr{A} \wedge X^{+}\right) \rightarrow E_{p, q}^{1}
$$

of right $\pi_{*}(\mathscr{A})$-modules.

By Theorem (3.12), the sequence

$$
0 \longleftarrow \pi_{*}(\mathscr{A} \wedge F) \stackrel{\partial_{0}}{\longleftarrow} R_{0, *} \stackrel{\partial_{1}}{\longleftarrow} R_{1, *} \longleftarrow \cdots
$$

is a free right $\pi_{*}(\mathscr{A} \wedge H)$-resolution of $\pi_{*}(\mathscr{A} \wedge F)$. The homomorphisms

are compositions

$$
d_{p, q}^{1}: E_{p, q}^{1} \rightarrow E_{p-1, q}^{1}
$$

$$
\begin{aligned}
\pi_{p+q+1}\left(\mathscr{Y}^{p} \wedge_{H} X^{+}, \mathscr{Y}^{p-1} \wedge_{H} X^{+}\right) & \stackrel{\partial}{\longrightarrow} \pi_{p+q}\left(\mathscr{Y}^{p-1} \wedge_{H} X^{+}\right) \\
& \longrightarrow \pi_{p+q}\left(\mathscr{Y}^{p-1} \wedge_{H} X^{+}, \mathscr{Y}^{p-2} \wedge_{H} X^{+}\right),
\end{aligned}
$$

which under the isomorphisms

$$
\pi_{p+q+1}\left(\mathscr{Y}^{p} \wedge_{H} X^{+}, \mathscr{Y}^{p-1} \wedge_{H} X^{+}\right) \stackrel{\cong}{\longrightarrow} \pi_{p+q+1}\left(\mathscr{X}^{p} \wedge_{H} X^{+}, \mathscr{X}^{p-1} \wedge_{H} X^{+}\right),
$$

coincide with the compositions

$$
\begin{aligned}
\pi_{p+q+1}\left(\mathscr{X}^{p} \wedge_{H} X^{+}, \mathscr{X}^{p-1} \wedge_{H} X^{+}\right) & \rightarrow \pi_{p+q}\left(\mathscr{X}^{p-1} \wedge_{H} X^{+}\right) \\
& \rightarrow \pi_{p+q}\left(\mathscr{X}^{p-1} \wedge_{H} X^{+}, \mathscr{X}^{p-2} \wedge_{H} X^{+}\right)
\end{aligned}
$$

By Proposition (2.39), the diagram

$$
\begin{aligned}
& R_{p, *} \underset{\pi .\left(\mathscr{A} \wedge H^{+}\right)}{\otimes} \pi_{*}\left(\mathscr{A} \wedge X^{+}\right) \stackrel{\gamma_{*}^{p}}{\longrightarrow} E_{p, *}^{1}
\end{aligned}
$$

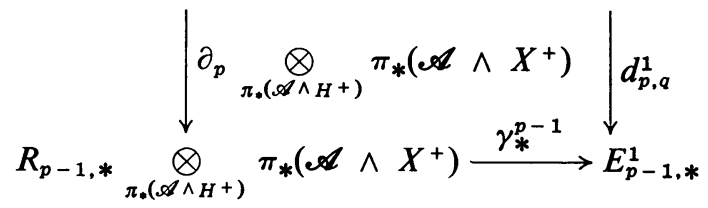

commutes, and by Corollary (2.42) it is a diagram of right $\pi_{*}(\mathscr{A})$-modules. The isomorphism

$$
E_{p, q}^{2} \cong \operatorname{Tor}_{p, q}^{h(H ; \mathscr{A})}\left(\tilde{h}_{*}(F ; \mathscr{A}), h_{*}(X ; \mathscr{A})\right)
$$

of right $\pi_{*}(\mathscr{A})$-modules follows. That this isomorphism has the naturality asserted in conclusion (6) is an immediate consequence of the fundamental theorem of homological algebra and of Theorem (3.10). 
We conclude this section by indicating how Theorem (4.3) may be obtained for $K O_{*}$ and $K U_{*}$, the homology theories associated to real and complex $K$-theory, respectively [2]. It will be necessary to assume that $X, H$ and $F$ have the homotopy type of CW-complexes. We let $K_{*}(X)$ stand for either $K O_{*}(X)$ or $K U_{*}(X)$.

It is not hard to show that $K O_{*}$ and $K U_{*}$ may be represented by ring spectra, that is, by spectra satisfying (1), (2) and (3) of Definition (2.7) up to homotopy. Theorem (4.3) is then proved for $K_{*}$ by replacing the functor $\pi_{*}$ by $K_{*}$ and setting $\mathscr{A}=\mathscr{S}$, the sphere spectrum. Notice that $K_{*}(\mathscr{S} \wedge X) \approx K_{*}(X)$.

In order to construct resolutions for $K_{*}$, one needs the following

Lemma (4.5). Let $\alpha \in K_{*}(X)$, where $X$ has the homotopy type of a CW-complex. Then there exists an integer $n$, a finite complex $Y$ with $K_{*}(Y)$ free over $K_{*}$ (point), an element $\beta \in K_{*}(Y)$ and a map $f: Y \rightarrow S^{n} X$ such that $f_{*}(\beta)=S^{n} \alpha$.

Proof. It suffices to prove (4.5) for $X$ a finite complex. For $K U_{*}$, this may be obtained by duality [14, Corollary (8.2)] from the dual statement for $K U^{*}$, proved by Atiyah in [1]. D. W. Anderson (private communication) has shown, using the spectral sequence of page 17 of [2] for $K O^{*}$, that the finite symplectic Grassmann manifolds $G_{m, n}(Q)$ have $K O^{*}\left(G_{m, n}(Q)\right)$ free over $K O^{*}$ (point). Atiyah's argument and duality now yield (4.5) for $K O_{*}$.

The one other result we need to adapt the proof of (4.3) to $K_{*}$ is

Lemma (4.6). Let $X$ and $Y$ be CW-complexes and let $K_{*}(X)$ be free over $K_{*}$ (point). Then

$$
K_{*}(X \times Y) \approx K_{*}(X) \underset{K_{*}(\text { point })}{\otimes} K_{*}(Y) .
$$

Proof. Let $\mathscr{A}$ be a ring spectrum representing $K_{*}$ and let $\Sigma$ be a basis for $K_{*}(X)$ over $K_{*}$ (point). Then an element $\sigma \in \Sigma$ is represented by a map

$$
f_{\sigma}: S^{n(\sigma)+k(\sigma)}=S_{\sigma} \rightarrow A_{k(\sigma)} \wedge X .
$$

Let $\mathscr{E}_{\sigma}$ be the spectrum with

$$
\begin{aligned}
E_{k(\sigma)+r} & =A_{r} \wedge S_{\sigma} & & \text { if } r \geqq 0 \\
& =* & & \text { if } r<0 .
\end{aligned}
$$

Define a map $f_{\sigma}: \mathscr{E}_{\sigma} \rightarrow \mathscr{A} \wedge X$ by means of the compositions

$$
A_{r} \wedge S_{\sigma} \stackrel{A_{r} \wedge f_{\sigma}}{\longrightarrow} A_{r} \wedge A_{k(\sigma)} \wedge X \stackrel{\mu \wedge X}{\longrightarrow} A_{r+k(\sigma)} \wedge X .
$$

Now let $\mathscr{E}_{\Sigma}$ be the wedge of the $\mathscr{E}_{\sigma}$ and let $f_{\Sigma}: \mathscr{E}_{\Sigma} \rightarrow \mathscr{A} \wedge X$ be the wedge of the $f_{\sigma}$. Since $K_{*}(X)=\pi_{*}(\mathscr{A} \wedge X)$ is free with basis $\Sigma$, it follows that

$$
f_{\Sigma *}: \pi_{*}\left(\mathscr{E}_{\Sigma}\right) \cong \pi_{*}(\mathscr{A} \wedge X)
$$

as modules over $\pi_{*}(\mathscr{A})=K_{*}$ (point). Since $Y$ is a CW-complex,

$$
\pi_{*}\left(\mathscr{E}_{\Sigma} \wedge Y\right) \stackrel{\cong}{\longrightarrow} \pi_{*}(\mathscr{A} \wedge X \wedge Y) .
$$

(4.6) now follows easily. 
REMARK. The isomorphism (4.7) shows the relation between Atiyah's method and ours.

V. Applications to fibrations. In this section we describe relations among fibrations from which the spectral sequences averred to in the introduction follow via Theorem (4.3). It is not our purpose in this paper to prove these relations. They can be deduced from results of Dold [6], Brown [5], and Stasheff [12] and are found explicitly in the setting and generality here considered in the book of Dyer and Eilenberg [7].

Recall that a map $p: X \rightarrow B$ is a fibration if there are lifting function $\Lambda^{p}: W^{p} \rightarrow$ $P X$ and delay function $\sigma^{p}: W^{p} \rightarrow R^{+}$satisfying certain relations (see $\S \mathrm{I}$ ), where $W^{p}=\{(x, \omega) \in X \times P B \mid p x=\omega 0\}$ with the subspace topology. Define

$$
p^{\prime}: W^{p} \rightarrow B
$$

by $p^{\prime}(x, \omega)=\eta_{\tau} \omega$, the terminal point of $\omega$. Then $p^{\prime}$ is a strong fibration with lifting function $\Lambda^{p^{\prime}}: W^{p^{\prime}} \rightarrow P W^{p}$ given by $\Lambda^{p^{\prime}}((x, \alpha), \beta)(r)=\left(x, \alpha+\beta_{0}^{t}\right)$, where $\beta_{0}^{t}$ is the path $\beta$ with domain restricted to $[0, t]$ and length $t$. We define maps

$$
\nu: W^{p} \rightarrow X \text { and } \mu: X \rightarrow W^{p}
$$

by $\nu(x, \omega)=\eta_{\tau} \Lambda^{p}(x, \omega)$ and $\mu x=\left(x, \varepsilon_{p x}\right)$, where $\varepsilon_{p x}$ is the path of zero length at $p x$. The maps $\nu$ and $\mu$ are maps over $B$ and there are homotopies over $B: X \simeq \nu \mu$ and $W^{p} \simeq \mu \nu$; i.e., $p^{\prime}$ and $p$ are fiber homotopy equivalent.

Define a map

$$
\lambda^{p^{\prime}}: W^{p^{\prime}} \rightarrow W^{p}
$$

by $\lambda^{p^{\prime}}=\eta_{\tau} \Lambda^{p^{\prime}}$. Then

$$
\lambda^{p^{\prime}}((x, \alpha), \beta+\gamma)=\lambda^{p^{\prime}}\left(\lambda^{p^{\prime}}((x, \alpha), \beta), \gamma\right) ;
$$

i.e., $\lambda^{p^{\prime}}$ is a transitive lifting function.

A transitive lifting function defines an associative, unitary right action of the space of loops of $B$ at a point $b \in B$ on the fiber $F^{\prime}=p^{\prime-1} b$ by

is $a=\lambda^{p^{\prime}} \mid F^{\prime} \times \Omega B$.

$$
a: F^{\prime} \times \Omega B \rightarrow F^{\prime}
$$

Let $P_{b} B=\{\alpha \in P B \mid \alpha 0=b\}$ with the subspace topology. There is a map

$$
\bar{j}: F^{\prime} \times P_{b} B \rightarrow W^{p}
$$

given by $j((x, \alpha), \beta)=(x, \alpha+\beta)$. This map is balanced over $\Omega B$ and so it induces

$$
j: F^{\prime} \times_{\Omega B} P_{b} B \rightarrow W^{p} .
$$

Since $\eta_{\tau}: P_{b} B \rightarrow B$ is a strong left $\Omega B$-fibration, $\hat{\eta}_{\tau}: F^{\prime} \times{ }_{\Omega B} P_{b} B \rightarrow B$ is a strong fibration (Proposition (1.7)). The map $j$ is map of fibrations $\hat{\eta}_{\tau}$ to $p^{\prime}$ over the identity on $B$ and $j \mid F^{\prime} \times_{\Omega B} \Omega B: F^{\prime} \times_{\Omega B} \Omega B \rightarrow F^{\prime}$ is a homeomorphism (see (1.3)). For $B$ pathwise connected this implies $\pi_{*}(j)$ is an isomorphism. If, in addition, $B$ is locally contractible in the large, then $j$ is a fiber homotopy equivalence. 
In summary, we have

Proposition (5.5). If $p: X \rightarrow B$ is a fibration, then there are strong fibrations $\hat{\eta}_{\tau}$ and $p^{\prime}$ and maps of fibrations

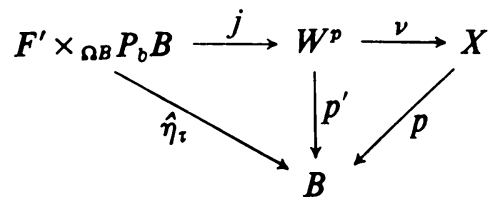

over the identity on $B$ such that

(1) $\nu$ is a fiber homotopy equivalence,

(2) if $B$ is pathwise connected, then $\pi_{*}(j)$ is an isomorphism, and

(3) if $B$ is also locally contractible in the large, then $j$ is a fiber homotopy equivalence.

Suppose $B$ is pathwise connected and locally contractible in the large and $p: X \rightarrow B$ is a fibration. Let $F=p^{-1} b$ denote the fiber at a point $b \in B$. Let $\mathscr{H}(F)$ denote the subspace of the space of maps of $F$ to $F$ which are homotopy equivalences. Let Prin $(p)$ denote the subspace of the space of maps of $F$ to $X$ of all maps $f$ such that $p f(F)$ is a point $b^{\prime}$ of $B$ and $f: F \rightarrow p^{-1} b^{\prime}$ is a homotopy equivalence, and let

$$
\hat{p}: \operatorname{Prin}(p) \rightarrow B
$$

be given by $\hat{p}(f)=p f(F)$. Then $\mathscr{H}(F)$ is a monoid and $\hat{p}$ is a principal left $\mathscr{H}(F)-$ bundle. Furthermore, there is a fiber homotopy equivalence

$$
k: F \times \mathscr{H}_{(F)} \operatorname{Prin}(p) \rightarrow X
$$

over the identity on $B$, (see [7]).

In the notation of Proposition (5.5), since $p^{\prime}: W^{p} \rightarrow B$ has a transitive lifting function, $\hat{p}^{\prime}: \operatorname{Prin}\left(p^{\prime}\right) \rightarrow B$ has a left $\mathscr{H}\left(F^{\prime}\right)$-equivariant transitive lifting function $\lambda$ given by

$$
\lambda(f, \omega)(x)=\lambda^{p^{\prime}}(f x, \omega)
$$

for $f: F^{\prime} \rightarrow p^{\prime-1} b^{\prime}$ and $\omega$ a path in $B$ with $\omega 0=b^{\prime}$. This function defines a map

$$
\bar{c}: \mathscr{H}\left(F^{\prime}\right) \times P_{b} B \rightarrow \operatorname{Prin}\left(p^{\prime}\right)
$$

by $\bar{c}(f, \omega)=\lambda(f, \omega)$. The map $\bar{c}$ is a left $\mathscr{H}\left(F^{\prime}\right)$-map and is balanced over $\Omega B$. It thus induces a map

$$
c: \mathscr{H}\left(F^{\prime}\right) \times{ }_{\Omega B} P_{b} B \rightarrow \operatorname{Prin}\left(p^{\prime}\right)
$$

of left $\mathscr{H}\left(F^{\prime}\right)$-bundles over the identity on $B$. The composition

$$
\begin{gathered}
F^{\prime} \times{ }_{\Omega B} P_{b} B \cong F^{\prime} \times \mathscr{H}\left(F^{\prime}\right) \\
\stackrel{\mathscr{H}\left(F^{\prime}\right) \times{ }_{\Omega B} P_{b} B}{F^{\prime} \times \mathscr{H}_{\left(F^{\prime}\right)} c} \longrightarrow F^{\prime} \times \mathscr{H ( F ^ { \prime } )} \operatorname{Prin}\left(p^{\prime}\right) \stackrel{k^{\prime}}{\longrightarrow} W^{p}
\end{gathered}
$$


is the map $j$ of Proposition (5.5) and each of $F^{\prime} \times \mathscr{H}_{\left(F^{\prime}\right)} C$ and $k^{\prime}$ is a fiber homotopy equivalence. This is

Proposition (5.10). If $B$ is pathwise connected and locally contractible in the large map $p: X \rightarrow B$ is a fibration, then the map $j$ of Proposition (5.5) factors

$$
F^{\prime} \times{ }_{\Omega B} P_{b} B \stackrel{F^{\prime} \times \mathscr{H}_{(F)} c}{\longrightarrow} F^{\prime} \times \mathscr{H}_{\left(F^{\prime}\right)} \operatorname{Prin}\left(p^{\prime}\right) \stackrel{k^{\prime}}{\longrightarrow} W^{p}
$$

through fiber homotopy equivalences with $P_{b} B \rightarrow \operatorname{Prin}\left(p^{\prime}\right)$ a map of left $\Omega B$-spaces and $\Omega B \rightarrow \mathscr{H}\left(F^{\prime}\right)$ a map of monoids.

If $G$ is a monoid each element of which has an inverse up to homotopy, $F$ is a right $G$-space, $B$ is a pathwise connected space which is locally contractible in the large, and $p: X \rightarrow B$ is the associated bundle $F \times{ }_{G} E$ to the principal left $G$-bundle $\bar{p}: E \rightarrow B$, then there exist a morphism of monoids $G \stackrel{\alpha}{\rightarrow} \mathscr{H}(F)$ given by $\alpha(g): F \rightarrow F$ is $\alpha(g) x=x g$ and a map $\tau: E \rightarrow \operatorname{Prin}(p)$ over $B$ of left $G$-spaces given by

$$
\tau(y): F \rightarrow \bar{p}^{\prime} \bar{p} y
$$

is $\tau(y)(x)=\left\{x \times_{G} y\right\}$. The composite.

$$
F \times_{G} E \stackrel{F \times_{G} \tau}{\longrightarrow} F \times_{G} \operatorname{Prin}(p) \stackrel{F \times_{\alpha} \operatorname{Prin}(p)}{\longrightarrow} F \times \mathscr{H}(F) \operatorname{Prin}(p)
$$

is a fiber homotopy equivalence over the identity on $B$.

Proposition (5.11). If $p: X \rightarrow B$ is the associated bundle with fiber $F$ to the principal left $G$-bundle $\bar{p}: E \rightarrow B$ and right action of $G$ on $F$, there are morphisms of monoids $G \stackrel{\alpha}{\rightarrow} \mathscr{H}(F)$ and of left $G$-spaces $E \stackrel{\tau}{\rightarrow}$ Prin $(p)$ over $B$ such that

$$
X=F \times_{G} E \stackrel{F \times_{\alpha} \tau}{\longrightarrow} F \times_{\mathscr{H}(F)} \operatorname{Prin}(p)
$$

is a fiber homotopy equivalence over $B$.

We summarize these results in

THEOREM (5.12). Let $\mathscr{A}$ be a strict ring spectrum and $p: X \rightarrow B$ a fibration. Then there is a right $h_{*}(\mathrm{pt}$; $)$ spectral sequence, ${ }_{1} E_{p, q}^{r}$ with limit associated with a filtration of $h_{*}(X ; \mathscr{A})$ and with

$$
{ }_{1} E_{p, q}^{2} \cong \operatorname{Tor}_{p, q}^{h \cdot(\Omega B ; \mathscr{A})}\left(h_{*}(F ; \mathscr{A}), h_{*}(\mathrm{pt} ; \mathscr{A})\right) .
$$

If $B$ is pathwise connected and locally contractible in the large, there is another such spectral sequence $E_{p, q}^{r}$ with limit associated with a filtration of $h_{*}(X ; \mathscr{A})$, with

$$
E_{p, q}^{2} \cong \operatorname{Tor}_{p, q}^{h,(\mathscr{H}(F) ; \mathscr{A})}\left(h_{*}(F ; \mathscr{A}), h_{*}(\operatorname{Prin}(p) ; \mathscr{A})\right),
$$

and with a morphism ${ }_{1} E^{r} \rightarrow E^{r}$ of right $h_{*}(\mathrm{pt} . ; \mathscr{A})$-spectral sequences which at the $E^{2}$-level under the isomorphisms on the $E^{2}$-terms is induced by a monoid morphism $\Omega B \rightarrow \mathscr{H}(F)$ and a map $P_{b} B \rightarrow$ Prin $(p)$ of left $\Omega B$-spaces. 
If also $p$ is the associated bundle to the principal left $G$-bundle $\bar{p}: E \rightarrow B$ with fiber the right $G$-space $F$, there is another such spectral sequence ${ }_{2} E_{p, q}^{r}$ with limit associated with a filtration of $h_{*}(X ; \mathscr{A})$, with

$$
{ }_{2} E_{p, q}^{2} \cong \operatorname{Tor}_{p, q}^{h ;(G ; \mathscr{A})}\left(h_{*}(F ; \mathscr{A}), h_{*}(E ; \mathscr{A})\right)
$$

and with a morphism ${ }_{2} E^{r} \rightarrow E^{r}$ of right $h_{*}$ (pt.; $\mathscr{A}$ )-spectral sequences which at the $E^{2}$-level under the isomorphisms on the $E^{2}$-terms is induced by a monoid morphism $G \rightarrow \mathscr{H}(F)$ and a map $E \rightarrow$ Prin ( $p$ ) of left G-spaces.

Proof. The proof of this theorem is essentially just a recapitulation of the previous three propositions and Theorem (4.3).

We indicate only the reduction from Proposition (5.5) to results from which the spectral sequence (5.13) is an immediate consequence of Theorem (4.3). The others are similarly obtained using Propositions (5.10) and (5.11).

In the notation of Proposition (5.5), we have

$$
\begin{aligned}
\pi_{*}\left(\mathscr{A} \wedge X^{+}\right) & \cong \pi_{*}\left(\mathscr{A} \wedge\left(F^{\prime} \times_{\Omega B} P_{b} B\right)^{+}\right) \\
& \cong \pi_{*}\left(\mathscr{A} \wedge\left(F^{\prime+} \wedge_{\Omega B} P_{b} B^{+}\right)\right) \\
& \cong \pi_{*}\left(\left(\mathscr{A} \wedge F^{+}\right) \wedge_{\Omega B} P_{b} B^{+}\right) .
\end{aligned}
$$

Then $F^{+}$is a $Q H^{\circ}$-space, $\eta_{\tau}: P_{b} B \rightarrow B$ is a principal left $\Omega B$-bundle, and Theorem (4.3) implies the conclusion (5.13).

EXAMPLES (5.16)(a). Let $G$ be a monoid for which there is a classifying space $B_{G}$. Then for $F$ a point, $X=F \times{ }_{G} E_{G}=B_{G}$. Thus, the spectral sequence (5.15) converges to quotients of a filtration of $h_{*}\left(B_{G} ; \mathscr{A}\right)$ and

$$
{ }_{2} E_{p, q}^{2} \cong \operatorname{Tor}_{p, q}^{h,(G ; \mathscr{A})}\left(h_{*}(\mathrm{pt} . ; \mathscr{A}), h_{*}\left(\mathrm{pt} ; \mathscr{A}^{\prime}\right)\right) \text {. }
$$

(b) Let $G=e, X=F \times B$ and $E=B$. Then (5.15) converges 'to quotients of a filtration of $h_{*}(F \times B ; \mathscr{A})$ and

$$
{ }_{2} E_{p, q}^{2} \cong \operatorname{Tor}_{p, q}^{h \cdot(\mathrm{pt} . ; \mathscr{A})}\left(h_{*}(F ; \mathscr{A}), h_{*}(B ; \mathscr{A})\right) .
$$

This was obtained earlier in [9].

At least one of the authors conjectures this implies Theorem (4.3).

\section{REFERENCES}

1. M. F. Atiyah, Vector bundles and the Künneth formula, Topology 1 (1962), 245-248.

2. M. F. Atiyah and F. Hirzebruch, Vector bundles and homogeneous spaces, Proc. Sympos. Pure Math. vol. 3, Amer. Math. Soc., Providence, R. I., 1961, pp. 7-38.

3. M. G. Barratt and P. J. Hilton, On join operations of homotopy groups, Proc. London Math. Soc. 3 (1953), 430-445.

4. A. L. Blakers and W. S. Massey, The homotopy groups of a triad. II, Ann. of Math. (2) 55 (1952), 192-201.

5. E. H. Brown, Jr., Twisted tensor products. I, Ann. of Math. (2) 69 (1959), 223-246.

6. A. Dold, Partitions of unity in the theory of fibrations, Ann. of Math. (2) 78 (1963), 223-255.

7. E. Dyer and S. Eilenberg (to appear). 
8. S. Eilenberg and S. MacLane, On the groups $H(\pi, n)$. I, Ann. of Math. (2) 58 (1953), 55-106.

9. D. S. Kahn, A Künneth theorem for some generalized homology theories (to appear).

10. J. Milnor, The geometric realization of a semisimplicial complex, Ann. of Math. (2) 65 (1957), 3.57-362.

11. E. Spanier, Quasi-topologies, Duke Math. J. 30 (1963), 1-14.

12. J. Stasheff, A classification theorem for fibre spaces, Topology 2 (1963), 239-246.

13. N. E. Steenrod, A convenient category of topological spaces, Michigan Math. J. 14 (1967), 133-152.

14. G. W. Whitehead, Generalized homology theories, Trans. Amer. Math. Soc., 102 (1962), 227-283.

City University of New York, Graduate Division, New York, New York

NORTHWESTERN UNIVERSITY,

Evanston, ILLinois 